\title{
On the punctual Hilbert scheme of a symplectic fourfold
}

\author{
Claire Voisin \\ Institut de mathématiques de Jussieu, CNRS,UMR 7586
}

Pour Herb, à l'occasion de

son soixantième anniversaire

\section{Introduction}

If $S$ is a smooth complex surface, the punctual Hilbert scheme (or more precisely Douady space, in the non algebraic case) $H_{i l b^{k}}(S)$, parametrizing 0-dimensional subschemes $Z \subset X$ of length $k$ is by Fogarty's theorem [7] a smooth complex variety, and the Hilbert-Chow morphism

$$
c: H i l b^{k}(S) \rightarrow S^{(k)}
$$

which to a subscheme associates its cycle, that is its support together with the local multiplicities, makes it a desingularization of the symmetric product $S^{(k)}$. The morphism $c$ is an isomorphism over the Zariski open set $S_{0}^{(k)}$ parametrizing $k$-uples of distinct points, so that $\operatorname{Hilb}^{k}(S)$ is as well a smooth partial compactification of $S_{0}^{(k)}$, which is compact when $S$ is compact. The fiber $c^{-1}(z), z \in S^{(k)}$ is a singular complex projective variety, the isomorphism class of which depends only on the multiplicities $n_{i}$ of the cycle $z=\sum_{i} n_{i} x_{i}, \sum_{i} n_{i}=k$, where the $x_{i}$ 's are distinct.

In the paper [16], we proposed two approaches for the construction of an analogue of the Hilbert scheme for any almost complex fourfold $(X, J)$, without any integrability assumption on $J$.

The first one worked only for the (open) part of the Hilbert scheme which parametrizes cycles $z$ which at each point of their support are either curvilinear, i.e contained in a smooth curve, or of multiplicity at most 3 , which includes the first infinitesimal neighbourhood of a point. Although limited to an open subset of the Hilbert scheme, this construction had the advantage of being canonical, that is to depend only on $J$. In fact there is an obvious notion of pseudoholomorphic subscheme, which is locally either curvilinear or the first infinitesimal of a point, and we had only to put a differentiable structure on the set of such objects.

The second construction provides us with a differentiable manifold $H i l b^{k}(X)$ of real dimension $4 k$, endowed with a continuous proper map

$$
c: \operatorname{Hilb}^{k}(X) \rightarrow X^{(k)}
$$

which is a diffeomorphism over the differentiable manifold $X_{0}^{(k)}$. More generally for each $z \in X^{(k)}$ the fiber $c^{-1}(z) \subset H_{i l b}(X)$ is a singular differentiable manifold, canonically diffeomorphic to the fiber $c_{z}^{-1}(z)$, where $c_{z}: H i l b^{k}\left(X_{z}\right) \rightarrow X_{z}^{(k)}$ is the Hilbert-Chow morphism relative to an integrable complex structure $I_{z}$ defined in a 
neighbourhood $X_{z}$ of $\operatorname{Supp} z \subset X$. Hence our Hilbert scheme is a desingularization of the symmetric product $X^{(k)}$, which has its fibers over $X^{(k)}$ as in the integrable case. It is easy to see that our construction coincides with the complex construction in the integrable case.

We show furthermore in that paper that $\operatorname{Hilb}^{k}(X)$ can be provided with a natural stable almost complex structure (that is a complex structure on the direct sum $T_{H i l b^{k}(X)} \oplus T$, where $T$ is a trivial bundle), and that it is well defined up to diffeomorphisms isotopic to the identity. Furthermore it depends only of the deformation class of $J$.

There is now a natural class of almost complex fourfolds, which is provided by the symplectic fourfolds : given such a pair $(X, \omega)$, Gromov [10] observes that the set of almost complex structures $J$ compatible with $\omega$, that is satisfying the conditions

$$
\omega(u, v)=\omega(J u, J v), u, v \in T_{X, x}, \omega(u, J u)>0,0 \neq u \in T_{X, x}
$$

is contractible. Applying the previous construction to any such almost complex structure provides us with a differentiable $2 k$-fold $H i l b^{k}(X)$, determined by $(X, \omega)$ up to diffeomorphisms isotopic to the identity. In the case where we can impose furthermore $J$ to be integrable, that is when $(X, J)$ is underlying a complex surface $S$ and $\omega$ is a Kähler form on $S$, we have the following result which is essentially proved in $[15]$

Theorem 1 Let $S$ be a compact Kähler surface. Then $H i l b^{k}(S)$ is a Kähler variety.

The cohomology in small degrees of the Hilbert scheme is described as follows (cf. [1]): let $E \subset H_{i l b}(S)$ be the exceptional divisor, $E=c^{-1}(\Delta)$, where $\Delta \subset S^{(k)}$ is the generalized diagonal. Then the divisor $E$ is 2-divisible in Pic Hilb ${ }^{k}(S)$, hence the class $c_{1}(E)$ is equal to $2 \delta, \delta \in H^{2}\left(H_{i l b}^{k}(S), \mathbb{Z}\right)$. We have

$$
H^{2}\left(\operatorname{Hilb}^{k}(S), \mathbb{Z}\right) \cong c^{*} H^{2}\left(S^{(k)}, \mathbb{Z}\right) \oplus \mathbb{Z} \delta .
$$

Now $H^{2}\left(S^{(k)}, \mathbb{R}\right) \cong H^{2}\left(S^{k}, \mathbb{R}\right)^{\text {inv }}$ contains naturally $H^{2}(S, \mathbb{R})$ by the map

$$
\alpha \mapsto \alpha_{k}=\sum_{i} p r_{i}^{*} \alpha \in H^{2}\left(S^{k}, \mathbb{R}\right)^{i n v}
$$

A more precise version of theorem 1 is then

Theorem 2 Let $\omega$ be a Kähler form on the compact surface $S$. For $0<\lambda$ sufficiently small, there is a Kähler metric of Kähler class $c^{*}[\omega]_{k}-\lambda \delta$ on Hilb ${ }^{k}(S)$.

Coming back to the symplectic or almost complex situation, one can show easily that the description of the cohomology of the almost complex Hilbert scheme is exactly the same as in the complex case (cf. [13], [8]). This follows from the existence of a stratification of $X^{(k)}$ with differentiable strata, and the fact that over each stratum the fibers of the Hilbert-Chow morphism are the same as in the integrable case. Hence starting with the symplectic class $[\omega] \in H^{2}(X, \mathbb{R})$ we get a class $c^{*}[\omega]_{k} \in H^{2}\left(H i l b^{k}(X), \mathbb{R}\right)$. Furthermore, the codimension 2 singular differentiable subvariety $E$ has a class $[E]=2 \delta, \delta \in H^{2}\left(H i l b^{k}(X), \mathbb{Z}\right)$. The main purpose of this paper is to prove the following symplectic analogue of theorem 2 
Theorem 3 Let $(X, \omega)$ be a compact symplectic fourfold. There exists a positive real number $\lambda_{0}$, such that for any $0<\lambda<\lambda_{0}$, there is a symplectic form on $H_{i l b}{ }^{k}(X)$ of cohomology class $c^{*}[\omega]_{k}-\lambda \delta$. Furthermore, all these symplectic forms belong to a well defined deformation class of symplectic forms on $H i l b^{k}(X)$.

The last section of this paper discusses potential applications of this result to the study of symplectic fourfolds. The first section reviews the construction of [16]. The two next sections are devoted to the construction of the symplectic form.

Acknowledgements. I would like to thank Jim Bryan and Jonathan Wahl who asked the question whether the manifolds constructed in [16] were symplectic.

\section{Review of the construction of $H_{i l b}^{k}(X)$}

We review in this section the construction of a punctual Hilbert scheme $\operatorname{Hilb}^{k}(X)$, for any $\mathcal{C}^{\infty}$ almost complex fourfold $(X, J)$.

Let

$$
Z \subset X^{(k)} \times X, Z=\{(z, x), x \in z\}
$$

be the incidence set. We shall construct a family of manifolds $H i l b_{I}^{k}(X)$ depending differentiably on a parameter $I$. The set of such data $I$ is contractible, hence the fact that all the manifolds $H i l b_{I}^{k}(X)$ are canonically diffeomorphic up to diffeomorphisms isotopic to the identity is a consequence of Ehresmann's theorem, at least in the compact case.

The auxiliary parameter $I$ is the data of a relative complex structure on an open neighbourhood $W \subset X^{(k)} \times X$ of the incidence set $Z$, where "relative" is relative with respect to the first projection $p r_{1}: W \rightarrow X^{(k)}$. That is, for each $z \in X^{(k)}$, we have a complex structure $I_{z}$ on a neighbourhood $W_{z}$ of $S u p p z$ in $X$, which should depend differentiably with $z$, if one puts on $X^{(k)}$ the quotient (singular) differentiable structure.

We then define $H_{i l b}^{k}(X)$ as follows : the relative complex structure $I$ makes $p r_{1}: W \rightarrow X^{(k)}$ a differentiable family of complex surfaces. We can then perform the construction of the Hilbert scheme in family, and by Fogarty we get a family of smooth complex $2 k$-folds

$$
\pi: H i l b_{I}^{k}\left(W / X^{(k)}\right)=\cup_{z \in X^{(k)}} H i l b_{I_{z}}^{k}\left(W_{z}\right) \rightarrow X^{(k)} .
$$

Furthermore we have the relative Hilbert-Chow morphism, with value in the relative symmetric product of $W$ over $X^{(k)}$

$$
c_{r e l}: H i l b_{I}^{k}\left(W / X^{(k)}\right) \rightarrow W^{(k) / X^{(k)}} .
$$

We note now that since $W \subset X^{(k)} \times X$, there is a natural (open) inclusion

$$
i: W^{(k) / X^{(k)}} \hookrightarrow X^{(k)} \times X^{(k)}
$$

which identifies $\pi$ with $p r_{1}$. We define now

$$
\operatorname{Hilb}_{I}^{k}(X):=\left(i \circ c_{r e l}\right)^{-1}(\text { Diag }),
$$


where Diag $\subset X^{(k)} \times X^{(k)}$ is the diagonal. The Hilbert-Chow map

$$
\operatorname{Hilb}_{I}^{k}(X) \rightarrow X^{(k)}
$$

is then defined by

$$
c=p r_{1} \circ i \circ c_{r e l}=p r_{2} \circ i \circ c_{r e l} \text {. }
$$

By construction, the fiber $c^{-1}(z)$ identifies to $c_{I_{z}}^{-1}(z)$ for $z \in X^{(k)}$, so that as anounced, our Hilbert scheme has its fibers over $X^{(k)}$ exactly as in the integrable case.

This definition makes $H i l b_{I}^{k}(X)$ only a topological space. Indeed there is a (singular) differentiable structure on $W^{(k) / X^{(k)}}$ for which $c_{r e l}$ is differentiable, but it does not coincide with the product differentiable structure given by $i$, hence Diag is not a differentiable subvariety for this differentiable structure. Furthermore, even in the integrable case, the Hilbert-Chow morphism $H i l b^{k}(S) \rightarrow S^{(k)}$ is not differentiable for the quotient differentiable structure on $S^{(k)}$, so we do not expect the differentiable structure on our $H_{i l b}^{k}(X)$ to be compatible with the quotient differentiable structure on $X^{(k)}$. We explain now what are the supplementary conditions we impose on $I$ in order to be able to put a differentiable structure on $H i l b b_{I}^{k}(X)$. These conditions are local triviality conditions on $I$ which are summarized in the next proposition.

Let $\tilde{Z} \subset X^{k} \times X$ be the incidence set, that is

$$
\tilde{Z}=\left\{\left(z_{1}, \ldots, z_{k}, z\right), \exists i, z=z_{i}\right\} .
$$

The relative complex structure $I$ will be induced by a relative complex structure on a neighbourhood $\tilde{W}$ of $\tilde{Z}$ in $X^{k} \times X$, invariant under the action of the symmetric group $\mathfrak{S}_{k}$ on the first factor.

For each partition $S=\left\{S_{1}, \ldots, S_{l}\right\}$ of $\{1, \ldots, k\}$, that is

$$
\{1, \ldots, k\}=\sqcup_{i} S_{i},
$$

let $\Delta_{S}$ be the diagonal indexed by $S$, that is $\Delta_{S}$ is the set of points $\left(x_{1}, \ldots, x_{k}\right) \in X^{k}$ such that $x_{i}=x_{j}$ if for some $\alpha$ the indices $i$ and $j$ belong to $S_{\alpha}$.

Proposition 1 [16] There exist a relative complex structure $\tilde{I}$ as above, and for each partition $S$ a differentiable retraction

$$
R_{S}: X^{k} \rightarrow \Delta_{S}
$$

defined in a neighbourhood of $\Delta_{S}$, satisfying the following properties :

1. Everything is compatible with the symmetric group action $: \forall \sigma \in \mathfrak{S}_{k}$, we have $(\sigma, i d)(\tilde{W})=\tilde{W}, \sigma^{*} \tilde{I}=\tilde{I}$ and $\sigma \circ R_{S} \circ \sigma^{-1}=R_{\sigma(S)}$.

2. The $R_{S}$ 's satisfy the condition

$$
\forall S^{\prime}, S \text { such that } \Delta_{S^{\prime}} \subset \Delta_{S}, R_{S^{\prime}} \circ R_{S}=R_{S^{\prime}}
$$

in a neighbourhood of $\Delta_{S^{\prime}}$.

3. In a neighbourhood of $\Delta_{S}$ the relative complex structure $\tilde{I}$ (which one sees locally as a family of complex structures on an open set of $X$ parametrized by $\left.X^{k}\right)$ is constant along the fibers of $R_{S}$. 
4. For each $z \in \Delta_{S}$, the fiber $R_{S}^{-1}(z) \subset X^{k}$ is a complex subvariety of $X^{k}$ endowed with the complex structure induced by $\tilde{I}_{z}$.

If $I$ is a relative complex structure on $W / X^{(k)}$ induced, by passing to the quotient, by a relative complex structure $\tilde{I}$ satisfying the properties above, we construct a differentiable structure on $H i l b_{I}^{k}(X)$ as follows. Let $z$ be a point of $X^{(k)}$ and let $\tilde{z} \in X^{k}$ be a point over $z$. There is a smallest diagonal $\Delta_{S}$ to which $\tilde{z}$ belongs, and denoting by $\mathfrak{S}_{S}$ the subgroup fixing pointwise $\Delta_{S}$, which is also the isotropy group of $\tilde{z}$, we have a local identification

$$
X^{(k)} \cong X^{k} / \mathfrak{S}_{S}
$$

Hence by the compatibility property 1 , the retraction $R_{S}$ will provide locally a retraction

$$
R: X^{(k)} \rightarrow \Delta
$$

defined near $z$, where $\Delta \cong \Delta_{S}$ is the image of $\Delta_{S}$ in $X^{(k)}$. (In the sequel we shall call $\Delta$ the minimal stratum or smooth stratum of $z$.) Next we know by property 3 that $I$ is constant along the fiber of $R$. Denoting $W_{\Delta}=W \cap \Delta \times X$ and $I_{\Delta}=I_{\mid \Delta}$ we have up to shrinking $W$ and over a neighbourhood of $z$ a diagram

$$
\begin{array}{cccc}
R \times I d: & W & \rightarrow & W_{\Delta} \\
& p r_{1} \downarrow & & p r_{1} \downarrow \\
R: & X^{(k)} & \rightarrow & \Delta
\end{array}
$$

and the fact that $I$ is constant along the fibers of $R$ means that this map is a holomorphic embedding along the fibers of $p r_{1}$. It follows that there is an induced morphism of the corresponding relative Hilbert schemes

$$
\begin{array}{cccc}
\tilde{R}: & H_{i l b^{k}}\left(W / X^{(k)}\right) & \rightarrow & H_{i l b}\left(W_{\Delta} / \Delta\right) \\
& \pi \downarrow & & \pi \downarrow \\
R: & X^{(k)} & \rightarrow & \Delta
\end{array}
$$

It is clear by the definition of $H i l b_{I}^{k}(X)$ as $c_{r e l}^{-1}$ (Diag) that the restriction of $\tilde{R}$ to $H_{i l b}^{k}(X)$ is a homeomorphism onto its image, which is equal to $\left(i \circ c_{r e l}\right)^{-1}\left(\Gamma_{R}\right)$, where

$$
c_{r e l}: \operatorname{Hilb}^{k}\left(W_{\Delta} / \Delta\right) \rightarrow W_{\Delta}^{(k) / \Delta}
$$

is the relative Hilbert-Chow morphism for the family of Hilbert schemes

$$
H_{i l b}^{k}\left(W_{\Delta} / \Delta\right) \rightarrow \Delta
$$

$i: W_{\Delta}^{(k) / \Delta} \hookrightarrow \Delta \times X^{(k)}$ is the natural inclusion induced by the open inclusion $W_{\Delta} \hookrightarrow \Delta \times X$, and $\Gamma_{R} \subset \Delta \times X^{(k)}$ is the graph of $R$. (More precisely all this is true over the considered neighbourhood $V$ of $z \in X^{(k)}$.) Notice that because $\Delta$ is smooth near $z, H i l b^{k}\left(W_{\Delta} / \Delta\right)$ is by Fogarty a smooth differentiable manifold. We show now that the image $\tilde{R}\left(H i l b_{I}^{k}(X) \cap c^{-1}(V)\right)$ is a differentiable smooth submanifold of $H_{i l b}{ }^{k}\left(W_{\Delta} / \Delta\right)$.

Indeed, consider the map

$$
\pi \circ \tilde{R}: \operatorname{Hilb}^{k}(X) \cap c^{-1}(V) \rightarrow \Delta .
$$


By what has been said above, its fiber over any $z^{\prime} \in \Delta \cap V$ identifies via $\tilde{R}$ to

$$
H i l b_{I_{z^{\prime}}}^{k}\left(W_{z^{\prime}}\right) \cap\left(R \circ c_{I_{z^{\prime}}}\right)^{-1}\left(z^{\prime}\right)=c_{I_{z^{\prime}}}^{-1}\left(R^{-1}\left(z^{\prime}\right)\right) .
$$

We use now the property 4 of proposition 1 . It says that $R^{-1}\left(z^{\prime}\right) \subset X^{(k)}$ is a complex analytic subspace of $X^{(k)}$ for the complex structure induced by $I_{z^{\prime}}$ which is the projection of an $\mathfrak{S}_{S}$-invariant smooth complex subvariety of $X^{k}$ transverse to the diagonal $\Delta_{S}$. It follows immediately from this that $c_{I_{z^{\prime}}}^{-1}\left(R^{-1}\left(z^{\prime}\right)\right) \subset H i l b_{z_{z^{\prime}}}^{k}\left(W_{z^{\prime}}\right)$ is a smooth complex subvariety of $H_{i l b} I_{z^{\prime}}\left(W_{z^{\prime}}\right)$. This complex subvariety varies differentiably with $z^{\prime} \in \Delta$ and this proves that $\tilde{R}\left(H i l b_{I}^{k}(X)\right) \subset H i l b^{k}\left(W_{\Delta} / \Delta\right)$ is a submanifold of $H_{i l b}^{k}\left(W_{\Delta} / \Delta\right)$.

This provides a differentiable structure on $H_{i l b}^{k}(X)$ over the considered neighbourhood of $z$. To show the compatibility of these differentiable structures, we use now the property 2 of proposition 1 . Indeed if two differentiable charts $\tilde{R}$ : $H_{i l b}(X) \hookrightarrow H i l b^{k}\left(W_{\Delta} / \Delta\right)$ and $\tilde{R}^{\prime}: H i l b^{k}(X) \hookrightarrow H i l b^{k}\left(W_{\Delta^{\prime}} / \Delta^{\prime}\right)$ as above overlap over an open set $U$ of $X^{(k)}$, we may assume that over this open set there is a third chart $\tilde{R}^{\prime \prime}: H i l b^{k}(X) \hookrightarrow H i l b^{k}\left(W_{\Delta^{\prime \prime}} / \Delta^{\prime \prime}\right)$ defined in $c^{-1}(U)$, with $\Delta^{\prime \prime} \subset \Delta$ and $\Delta^{\prime \prime} \subset \Delta^{\prime}$. Hence it suffices to prove the compatibility when $\Delta^{\prime} \subset \Delta$. But then, because the complex structures $I_{\Delta^{\prime}}$ and $I_{\Delta}$ are related by $I_{\Delta}=R^{\prime *} I_{\Delta^{\prime}}$, there is a differentiable map, which is even a submersion, of smooth manifolds

$$
\tilde{R}^{\prime}: H i l b^{k}\left(W_{\Delta} / \Delta\right) \rightarrow H i l b^{k}\left(W_{\Delta^{\prime}} / \Delta^{\prime}\right)
$$

which by definition sends $\tilde{R}\left(H i l b^{k}(X)\right) \subset H i l b^{k}\left(W_{\Delta} / \Delta\right)$ onto $\tilde{R}^{\prime}\left(H i l b^{k}(X)\right) \subset$ $H_{i l b}\left(W_{\Delta^{\prime}} / \Delta^{\prime}\right)$. It is immediate to show that this map restricted to

$$
\tilde{R}\left(H i l b^{k}(X)\right) \subset H i l b^{k}\left(W_{\Delta} / \Delta\right)
$$

is an immersion, hence a diffeomorphism onto its image. This concludes the construction of the differentiable structure on $\operatorname{Hilb}_{I}^{k}(X)$.

Remark 1 We already mentioned the fact that $c: H_{i l b}(X) \rightarrow X^{(k)}$ is not a differentiable map when $X^{(k)}$ is provided with the quotient differentiable structure, which means that in general for a differentiable function $f$ on $X^{(k)}$ its pull-back $f \circ c$ will not be differentiable on $H_{i l b}^{k}(X)$. Our construction of the local charts for Hilb ${ }_{I}^{k}(X)$ shows however that $f \circ c$ is differentiable if the pull-back $\tilde{f}$ of $f$ to $X^{k}$ factors through the retractions $R_{S}$ near the diagonals $\Delta_{S}$, or equivalently if $f$ factors locally through the retractions $R: X^{(k)} \rightarrow \Delta$ on the smooth stratum.

\section{Constructing the symplectic form}

Recall that $H i l b b_{I}^{k}(X)$ is defined as a closed subset of the relative Hilbert scheme $H i l b_{I}^{k}\left(W / X^{(k)}\right)$ and that the composed map

$$
c: \operatorname{Hilb}_{I}^{k}(X) \hookrightarrow \operatorname{Hilb}_{I}^{k}\left(W / X^{(k)}\right) \stackrel{\pi}{\rightarrow} X^{(k)}
$$

has for fiber $c^{-1}(z)$ a complex singular subvariety of $H i l b_{I_{z}}^{k}\left(W_{z}\right)$. We would like to construct a symplectic form on $H i l b^{k}(X)$ by combining the restriction of a relative Kähler form on $H i l b_{I}^{k}\left(W / X^{(k)}\right)$, and the pull-back $c^{*} \omega_{k}$ of the "symplectic form" $\omega_{k}$ 
on $X^{(k)}$ given by the form $\sum_{i} p r_{i}^{*} \omega$ on $X^{k}$, which is invariant under the symmetric group $\mathfrak{S}_{k}$. However, since the map $c$ is not differentiable for the quotient differentiable structure on the right, neither the pull-back of a relative Kähler form, nor the pull-back of $\omega$ will be differentiable forms on $\mathrm{Hilb}^{k}(X)$, so that in order to apply this construction, we have to regularize first both forms. The regularization process will be obtained using the following proposition, which will be proven (in a much more precise version) in the next section.

Proposition 2 There exists a differentiable map $\tilde{\phi}: X^{k} \rightarrow X^{k}$, which satisfies the following properties

1. $\tilde{\phi}$ commutes with the action of the symmetric group $\mathfrak{S}_{k}$ on $X^{k}$.

2. $\tilde{\phi}$ is close to the identity, so that $(\tilde{\phi}, i d)(\tilde{Z}) \subset \tilde{W}$, and $\tilde{\phi}^{*} \tilde{I}=\tilde{I}$ near $\tilde{Z}$.

3. Locally near each $\Delta_{S}$, $\tilde{\phi}$ takes value in $\Delta_{S}$ and factors through $R_{S}$, that is $\tilde{\phi}=\psi \circ R_{S}$ near $\Delta_{S}$, for some differentiable map $\psi: \Delta_{S} \rightarrow \Delta_{S}$.

Assuming this, we explain how to construct 2-forms on $H i l b_{I}^{k}(X)$. Let $\phi: X^{(k)} \rightarrow$ $X^{(k)}$ be the map induced by $\tilde{\phi}$ using the $\mathfrak{S}_{k}$-invariance. We have the following lemma

Lemma 1 The map $\phi \circ c: H i l b_{I}^{k}(X) \rightarrow X^{(k)}$ is differentiable with respect to the quotient differentiable structure on the right.

Notice that if $j: H i l b_{I}^{k}(X) \hookrightarrow H i l b_{I}^{k}\left(W / X^{(k)}\right)$ is the natural embedding, we have by construction $c=\pi \circ j$, where $\pi: H i l b_{I}^{k}\left(W / X^{(k)}\right) \rightarrow X^{(k)}$ is the structural map.

Proof. This follows from property 3 of proposition 2. It says that $\phi$ factors locally near each $z$ through the local retraction $R$ onto the smooth stratum of $z$, introduced in the previous section. Hence the result follows from the remark 1.

We will need also the following lemma

Lemma 2 Up to shrinking the open neighbourhood $W$ of $Z$ in $X^{(k)} \times X$, there exists for sufficiently large $N$ a differentiable immersion over $X^{(k)}$

$$
h: W \hookrightarrow X^{(k)} \times \mathbb{C}^{N}
$$

which is holomorphic on the fibers, for the relative complex structure I on the left.

Proof. The statement is local over $X^{(k)}$ since such an immersion is given by complex valued differentiable functions which are $I$-holomorphic along the fibers : such functions defined locally can be extended after multiplication by functions with small support on $X^{(k)}$, so that using a partition of unity on $X^{(k)}$ we can glue such local immersions to a global one.

As for the local situation, this is just the Newlander-Nirenberg theorem with parameters, which says that we can find local holomorphic coordinates on $W_{z}$ defined near $z$ and depending differentiably on the parameter $z \in \Delta$. (The fact that $X^{(k)}$ is singular here is not important: we simply write locally $X^{(k)}$ near $z$ as the quotient of $X^{k}$ by the isotropy group $\mathfrak{S}_{\tilde{z}}$ of $\tilde{z}$, and consider local relative holomorphic coordinates $\phi_{1}, \phi_{2}$ on $\tilde{W}_{\tilde{z}}$, defined near $\operatorname{Supp} z$ and varying differentiably with $\tilde{z} \in X^{k}$. Then clearly

$$
\sum_{\sigma \in \mathfrak{S}_{\tilde{z}}} \sigma^{*} \phi_{i}
$$


will also provide local relative holomorphic coordinates $\phi_{1}, \phi_{2}$ on $W_{z}$, defined near Supp $z$ and varying differentiably with $z \in X^{(k)}$.)

We fix now a Kähler form $\eta$ on $H_{i l b}\left(\mathbb{C}^{N}\right)$. (Of course $H i l b^{k}\left(\mathbb{C}^{N}\right)$ is singular, but by this we mean the restriction of a Kähler form on some projective space in which $\operatorname{Hilb}^{k}\left(\mathbb{C}^{N}\right)$ is holomorphically immersed, which exists since $H i l b^{k}\left(\mathbb{C}^{N}\right)$ is a quasi-projective variety.) The map $h: W \rightarrow X^{(k)} \times \mathbb{C}^{N}$ which is holomorphic on fibers over $X^{(k)}$ induces a differentiable map

$$
\tilde{h}: H i l b^{k}\left(W / X^{(k)}\right) \rightarrow X^{(k)} \times H i l b^{k}\left(\mathbb{C}^{N}\right) .
$$

The pull-back

$$
\eta^{\prime}:=\left(p r_{2} \circ \tilde{h}\right)^{*} \eta
$$

is a differentiable closed 2-form on $\operatorname{Hilb}_{I}^{k}\left(W / X^{(k)}\right)$, which restricts to a Kähler form on each fiber $H i l b_{I_{z}}^{k}\left(W_{z}\right)$ of $\pi: H i l b{ }_{I}^{k}\left(W / X^{(k)}\right) \rightarrow X^{(k)}$.

Next consider the differentiable map $\phi: X^{(k)} \rightarrow X^{(k)}$ introduced above. Since $\phi$ is close to the identity, and $\phi^{*} I=I$, we can shrink $W$ to $W^{\prime}$ so that $(\phi, i d)\left(W^{\prime}\right) \subset W$ and the map $(\phi, i d): W^{\prime} \rightarrow W$ is holomorphic on each fiber $W_{z}^{\prime}$ relative to the complex structure $I_{z}$. Hence there is a commutative diagram of families of complex varieties

$$
\begin{aligned}
& \Phi: \quad H i l b^{k}\left(W^{\prime} / X^{(k)}\right) \quad \rightarrow \quad H i l b^{k}\left(W / X^{(k)}\right) \\
& \pi \downarrow \\
& \phi: \quad X^{(k)} \quad \rightarrow \quad X^{(k)}
\end{aligned}
$$

and it follows that $\Phi^{*} \eta^{\prime}$ is also a relative Kähler form on $H i l b^{k}\left(W / X^{(k)}\right)$. We have now, recalling that $H i l b_{I}^{k}(X)$ is naturally contained in $H i l b^{k}\left(W^{\prime} / X^{(k)}\right)$ :

Lemma 3 The restriction $\Psi:=\Phi^{*} \eta_{\mid H_{i l b}^{k}(X)}^{\prime}$ is a differentiable (closed) 2-form.

Proof. This follows immediately from the commutative diagram (3.1), from the fact that $\eta^{\prime}=\left(p r_{2} \circ \tilde{h}\right)^{*} \eta$ and from lemma 1, which implies that the map

$p r_{2} \circ \tilde{h} \circ \Phi_{\mid H i l b^{k}(X)}: H i l b_{I}^{k}(X) \subset H i l b^{k}\left(W^{\prime} / X^{(k)}\right) \stackrel{\Phi}{\rightarrow} H i l b^{k}\left(W / X^{(k)}\right) \stackrel{p r_{2} \circ \tilde{h}}{\rightarrow} H i l b^{k}\left(\mathbb{C}^{N}\right)$

is differentiable. (By this we mean that its composition with any holomorphic embedding of $H i l b^{k}\left(\mathbb{C}^{N}\right)$ in a projective space is differentiable.)

Next let $\omega$ be the symplectic 2-form on $X$. The invariant symplectic form $\sum_{i} p r_{i}^{*} \omega$ on $X^{k}$ descends to a symplectic form $\omega_{k}$ on the open set $X_{0}^{(k)}$. We have now

Lemma 4 The differentiable closed 2-form $(\phi \circ c)^{*} \omega_{k}$ defined over the open set $(\phi \circ$ $c)^{-1}\left(X_{0}^{(k)}\right)$ of Hilb ${ }^{k}(X)$ extends naturally to a smooth closed 2 -form $\chi$ on Hilb ${ }^{k}(X)$.

Proof. Consider the 2-form $\tilde{\phi}^{*}\left(\sum_{i} p r_{i}^{*} \omega\right)$ on $X^{k}$. It is invariant under the symmetric group. Because $\tilde{\phi}$ factors locally near $z \in X^{k}$ through the retraction $R_{S}$, where $\Delta_{S}$ is the minimal diagonal containing $z$, we have locally

$$
\tilde{\phi}^{*}\left(\sum_{i} p r_{i}^{*} \omega\right)=R_{S}^{*} \mu
$$


where $\mu$ is a closed 2 -form on $\Delta_{S}$ defined near $z$. But we know that the map $R \circ c: \operatorname{Hilb}_{I}^{k}(X) \rightarrow \Delta$ is differentiable, where $R: X^{(k)} \rightarrow \Delta$ is the local factorisation of $\Delta_{S}$ through $\mathfrak{S}_{S}$. Hence the differential form $(R \circ c)^{*} \mu$ is a closed differential 2form defined over an open set of $\operatorname{Hilb}^{k}(X)$. It is easy to see that all these differential forms coincide on the intersections of such open sets to give the desired extension of $(\phi \circ c)^{*} \omega_{k}$.

The symplectic form we want to construct on $H_{i l b}^{k}(X)$ will be of the form

$$
\Omega=\chi+\lambda \Psi
$$

for $\lambda>0$ sufficiently small. In order that such form be everywhere nondegenerate we have to impose supplementary conditions to $\phi$, which will be explained in the proof of proposition 2. We will then prove in the next section

Theorem 4 The map $\phi$ being constructed as in the proof of proposition 2, there exists a positive real number $\lambda_{0}$ such that the form $\Omega=\chi+\lambda \Psi$ is a symplectic form on $\operatorname{Hilb}_{I}^{k}(X)$, for any $0<\lambda<\lambda_{0}$.

\section{Proof of proposition 2 and theorem 4}

Proof of Proposition 2. We recall first how the retractions $R_{S}: X^{k} \rightarrow \Delta_{S}$ defined in a neighbourhood of each diagonal $\Delta_{S}$ are constructed. These retractions come from tubular neighbourhoods

$$
i_{S}: N_{\Delta_{S} / X^{k}} \cong X^{k}
$$

defined in a neighbourhood of the zero section of the normal bundle of $\Delta_{S}$ in $X^{k}$. The retraction $R_{S}$ identifies via $i_{S}$ to the structural map $N_{\Delta_{S} / X^{k}} \rightarrow \Delta_{S}$.

The diffeomorphisms $i_{S}$ are constructed starting from the following data : let $p r_{j}: \Delta_{S} \rightarrow X$ be the $j$-th projection, and let $\Gamma_{j} \subset \Delta_{S} \times X$ be the graph of $p r_{j}$. Assume given a diffeomorphism over $\Delta_{S}$, defined in the neighbourhood of the 0 -section

$$
\psi_{j}: p r_{j}^{*} T_{X} \rightarrow \Delta_{S} \times X
$$

We suppose that for fixed $z \in \Delta_{S}$, the induced diffeomorphism $\psi_{j}(z): T_{X, z_{j}} \cong X$ defined near $0 \in T_{X, z_{j}}$ coincides with $\psi_{k}(z)$ if $z_{j}=z_{k}$. Furthermore, its differential at 0 should be the identity of $T_{X, z_{j}}$.

The diffeomorphisms $\psi_{j}$ provide now a diffeomorphism over $\Delta_{S}$

$$
\psi=\left(\psi_{j}\right): T_{X^{k} \mid \Delta_{S}} \cong \Delta_{S} \times X^{k} .
$$

The diffeomorphism $i_{S}$ is then obtained by restricting $\psi$ to $N_{\Delta_{S} / X^{k}}$, which is naturally contained in $T_{X^{k} \mid \Delta_{S}}$ as the kernel of the linear projection

$$
\pi_{S}: T_{X^{k} \mid \Delta_{S}} \rightarrow T_{\Delta_{S}}
$$

onto the space of invariants under $\mathfrak{S}_{S}$.

The relative complex structure $\tilde{I}_{S}$ on some neighbourhood $\tilde{W}_{S}$ of the incidence set (i.e the union of the graphs $\Gamma_{j}$ ) in $\Delta_{S} \times X$ is defined by choosing for each $j$ a 
complex structure $J_{j}$ on the vector bundle $p r_{j}^{*} T_{X}$ (it is here that the existence of an almost complex structure on $X$ plays a role), assuming again that the complex structures $J_{j}(z)$ and $J_{k}(z)$ on $T_{X, z_{j}}=T_{X, z_{k}}$ coincide whenever $z_{j}=z_{k}$.

Then we define the complex structure $\tilde{I}_{S}(z)$ (defined on a neighbourhood of Supp $z$ in $X$ ) to be induced in the neighbourhood of $z_{j} \in S u p p z$ by the diffeomorphism $\psi_{j}(z)$ and by the complex structure $J_{j}(z)$ on $T_{X, z_{j}}$. The relative complex structure $\tilde{I}$ defined on some neighbourhood $\tilde{W}$ of the incidence set $\tilde{Z} \subset X^{k} \times X$ will be equal to $R_{S}^{*} \tilde{I}_{S}$ near $\Delta_{S}$.

It is then immediate to show that the retraction $R_{S}$ and the relative complex structure $\tilde{I}$ defined near $\Delta_{S}$ constructed as above satisfy the property (essential for our construction) that the fibers $R_{S}^{-1}(z)$ are complex subvarieties (obviously transverse to $\Delta_{S}$ ) of $X^{k}$ for the complex structure on $X^{k}$ (defined near $z$ ) induced by $\tilde{I}_{z}$. Indeed, via the diffeomorphism $\psi_{z}: T_{X^{k}, z} \cong X^{k}$, this complex structure identifies to the constant complex structure on $T_{X^{k}, z}$ given by the $J_{j}(z)$ 's, and the fiber $R_{S}^{-1}(z)$ identifies to the complex vector subspace $\operatorname{Ker} \pi_{S}$.

The property $R_{S^{\prime}} \circ R_{S}=R_{S^{\prime}}$ near $\Delta_{S^{\prime}}$ when $\Delta_{S^{\prime}} \subset \Delta_{S}$ is translated as follows: near $\Delta_{S^{\prime}}$ the diffeomorphism

$$
i_{S^{\prime}}: N_{\Delta_{S^{\prime}} / X^{k}} \cong X^{k}
$$

sends the vector subbundle $N_{\Delta_{S^{\prime}} / \Delta_{S}}$ onto the diagonal $\Delta_{S}$. Because there is a natural splitting

$$
N_{\Delta_{S^{\prime}} / X^{k}}=N_{\Delta_{S^{\prime}} / \Delta_{S}} \oplus N_{\Delta_{S} / X^{k}}{ }_{\mid \Delta_{S^{\prime}}}
$$

given by the linear projection onto the space of invariants under $\mathfrak{S}_{S}$, there is a natural tubular neighbourhood of $N_{\Delta_{S^{\prime}} / \Delta_{S}}$ in $N_{\Delta_{S^{\prime}} / X^{k}}$, hence via $i_{S^{\prime}}$ we get an induced tubular neighbourhood of $\Delta_{S}$ in $X^{k}$ defined near $\Delta_{S}^{\prime}$. We ask that it coincides there with $i_{S}$. This condition is not satisfied if one takes for $\psi_{j}$ the exponential map with respect to some fixed metric on $X$. We can take for $\psi_{j}$ the exponential map away from the smaller diagonal contained in $\Delta_{S}$, but we have to modify it near the smaller diagonals. Of course everything is supposed to be equivariant with respect to the action of the symmetric group.

We explain now how we shall construct the map $\tilde{\phi}$ using these tubular neighbourhoods. We choose for each $S$ a metric $h_{S}$ on the vector bundle $N_{\Delta_{S} / X^{k}}$, compatible with the symmetric group action in the obvious way, and we ask that near $\Delta_{S^{\prime}} \subset \Delta_{S}$, $h_{S}$ is induced by $h_{S^{\prime}}$, by the tubular isomorphism $i_{S^{\prime}}$ and by the linear invariant projection

$$
N_{\Delta_{S^{\prime}} / X^{k}} \rightarrow N_{\Delta_{S} / X^{k} \mid \Delta_{S^{\prime}}} \cong \Delta_{S}
$$

We consider now the open sets

$$
\begin{gathered}
U_{S}=\left\{u \in N_{\Delta_{S} / X^{k}}, h_{S}(u)<\frac{\eta_{S}}{2}\right\}, \\
V_{S}=\left\{u \in N_{\Delta_{S} / X^{k}}, h_{S}(u)<\eta_{S}\right\},
\end{gathered}
$$

where the $\eta_{S}$ are sufficiently small positive real numbers. We then see these open sets as open sets of $X^{k}$.

Let now $\mu: \mathbb{R}^{+} \rightarrow \mathbb{R}^{+}$be a $\mathcal{C}^{\infty}$ function satisfying

$$
\mu(t)=0, t \leq \frac{1}{2}, \mu(t)=1, t \geq 1 .
$$


We define then $\phi_{S}: X^{k} \rightarrow X^{k}$ to be the identity outside $V_{S}$ and to be given by the formula

$$
\phi_{S}(u)=\mu\left(\frac{1}{\eta_{S}} h_{S}(u)\right) u
$$

in $V_{S}$. We note that $\phi_{S}$ identifies to $R_{S}$ in $U_{S}$. Furthermore $\phi_{S}$ satisfies the following compatibility condition with the action of $\mathfrak{S}_{k}$

$$
\sigma \circ \phi_{S} \circ \sigma^{-1}=\phi_{\sigma(S)}, \forall \sigma \in \mathfrak{S}_{k} .
$$

We suppose now the $\eta_{S}$ have been choosen so that

$$
V_{S} \cap V_{S^{\prime}} \subset U_{S \cdot S^{\prime}}
$$

if $\Delta_{S} \not \subset \Delta_{S^{\prime}}, \Delta_{S^{\prime}} \not \subset \Delta_{S}$, where $S \cdot S^{\prime}$ is the partition (or equivalence relation) generated by $S$ and $S^{\prime}$, so that we have

$$
\Delta_{S \cdot S^{\prime}}=\Delta_{S} \cap \Delta_{S^{\prime}}
$$

Indeed it is clear that this will be true once $\eta_{S} \ll \eta_{S^{\prime}}$ for any pair of diagonals such that $\Delta_{S^{\prime}} \subsetneq \Delta_{S}$.

It then follows that we have $\phi_{S \cdot S^{\prime}}=R_{S \cdot S^{\prime}}$ at a point $z$ such that $\phi_{S}(z) \neq z$ and $\phi_{S^{\prime}}(z) \neq z$, when none of the diagonals $\Delta_{S}$ and $\Delta_{S^{\prime}}$ is contained in the other.

We then define $\tilde{\phi}$ as follows : let $z \in X^{k}$ and suppose that $z \notin U_{S}$ for any $S$. Then it follows from (4.5) that the set of partitions

$$
W_{z}:=\left\{S, x \in V_{S}\right\}
$$

is totally ordered by the inclusion of the corresponding diagonals

$$
W_{z}=\left\{\Delta_{S_{0}} \subset \ldots \Delta_{S_{l}}\right\} .
$$

We then define

$$
\tilde{\phi}(z)=\phi_{S_{0}} \circ \phi_{S_{1}} \ldots \circ \phi_{S_{l}}(z) .
$$

More generally, consider the set of partitions

$$
W_{z}^{\prime}:=\left\{S, x \in U_{S}\right\}
$$

It has a minimal element $S_{z}$ by property (4.5). We introduce now the set of partitions

$$
W_{z}:=\left\{S, \Delta_{S} \subset \Delta_{S_{z}}, x \in V_{S}\right\} .
$$

By the minimality property of $S_{z}$ and by property (4.5), this set is totally ordered by the inclusion of the corresponding diagonals

$$
W_{z}=\left\{\Delta_{S_{0}} \subset \ldots \Delta_{S_{l}}\right\}
$$

and we then define

$$
\tilde{\phi}(z)=\phi_{S_{0}} \circ \phi_{S_{1}} \ldots \circ \phi_{S_{l}}(z) \circ R_{S_{z}}(z) .
$$

One checks easily that the map $\tilde{\phi}: X^{k} \rightarrow X^{k}$ so constructed is differentiable and it is clear by definition that it factors locally through the retractions $R_{S}$. Furthermore, since each $\phi_{S}$ preserves the relative complex structure $\tilde{I}$, (because it preserves the fibers of $R_{S}$ along which $\tilde{I}$ is constant, ) the same is true of $\tilde{\phi}$. The fact that $\tilde{\phi}$ commutes with the action of the symmetric group follows from the relation (4.4). Hence proposition 2 is proven. 
In order to prove theorem 4 we will need to impose supplementary conditions to our tubular neighbourhoods $i_{S}$ and to our metrics $h_{S}$, in order to take into account the symplectic structure of $X$.

First of all we ask that the local diffeomorphisms

$$
\psi_{j}: p r_{j}^{*} T_{X} \rightarrow \Delta_{S} \times X
$$

introduced in (4.2) pull-back the symplectic form $\omega$ on $X$ to a 2-form on the vector bundle $p r_{j}^{*} T_{X}$ which is the constant symplectic form $\omega_{z_{j}}$ on each fiber $T_{X, z_{j}}$.

Furthermore we ask that the complex structure $J_{j}$ on the vector bundle $p r_{j}^{*} T_{X}$ is compatible with the symplectic form induced by $\psi_{j}^{*} \omega$ on the fibers. This 2 -form together with the complex structure $J_{j}$ determine then a hermitian metric on $p r_{j}^{*} T_{X}$. We will choose then as metric $h_{S}$ the restriction to the subbundle $N_{\Delta_{S} / X^{k}} \subset T_{X^{k} \mid \Delta_{S}}$ of the product of these hermitian metrics on $T_{X^{k} \mid \Delta_{S}} \cong \Pi_{j} p r_{j}^{*} T_{X}$.

Now by definition, via the diffeomorphism

$$
\psi_{z}=\left(\psi_{j, z}\right): T_{X^{k}, z} \cong X^{k}
$$

the complex structure induced by $\tilde{I}_{S}(z)$ on $X^{k}$ near $z$ coincides with the complex structure $\oplus J_{j, z}$ on $\Pi_{j} T_{X, z_{j}}$; furthermore, by construction the 2 -form $\psi_{z}^{*}\left(\sum_{j} p r_{j}^{*} \omega\right)$ restricts to a constant form on the vector space $T_{X^{k}, z}$, and the fiber $R_{S}^{-1}(z)$ is the image under $\psi_{z}$ of the vector subspace $N_{\Delta_{S} / X^{k}, z}$. In conclusion, $X^{k}$ endowed with the complex structure induced on $X^{k}$ near $z$ by $\tilde{I}_{z}$, and the 2 -form $\sum_{j} p r_{j}^{*} \omega$ is a Kähler variety and the fiber $R_{S}^{-1}(z) \subset X^{k}$ is a Kähler subvariety of it. We shall prove the following version of theorem 4

Theorem 5 If $\tilde{\phi}$ is constructed as above, the metrics $h_{S}$ are the Kähler metrics and the $\eta_{S}$ are adequately choosen, (namely $\eta_{S} \ll \eta_{S^{\prime}}$ when $\Delta_{S^{\prime}} \subsetneq \Delta_{S}$,) there exists a positive real number $\lambda_{0}$ such that the form

$$
\Omega=\chi+\lambda \Psi
$$

is a symplectic form on $H i l b_{I}^{k}(X)$ for any $0<\lambda<\lambda_{0}$.

The end of this section is devoted to the proof of this theorem. We note to start with that this is in fact a local statement, by compactnesss of $H_{i l b}^{k}(X)$.

We note now the following lemma : let $u \in H i l b b_{I}^{k}(X)$; denote by $K_{u}$ the kernel

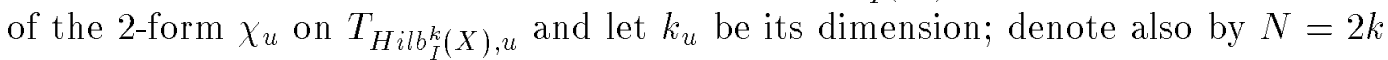
the complex dimension of $H_{i l b}^{k}(X)$. Then

Lemma 5 In order to prove that there exists a neighbourhood $U$ of $u$ and a real number $\lambda_{0}$ such the form $\Omega=\chi+\lambda \Psi$ is non degenerate on $U$ for $0<\lambda<\lambda_{0}$, it suffices to check the following.

1. We have $\chi^{N-k_{u}} \Psi^{k_{u}}>0$.

2. For any integer $l$ such $0 \leq l \leq k_{u}$, we have $\chi^{N-l} \Psi^{l} \geq 0$ in a neighbourhood of $u$. 
Here the sign of the $2 N$-forms $\chi^{N-l} \Psi^{l}$ is computed with respect to the canonical orientation (compatible with the stable almost complex structure) of $H i l b_{I}^{k}(X)$. In the local charts

$$
\operatorname{Hilb}_{I}^{k}(X) \stackrel{\operatorname{Roc}}{\rightarrow} \Delta
$$

which make $H i l b^{k}(X)$ a family of complex manifolds parametrized by $\Delta$, this orientation is induced by the complex orientation on the fibers and the symplectic orientation on the basis $\Delta$, which is an open set of some $X^{r}$.

Proof. Consider the function

$$
f(\lambda, u)=\frac{(\lambda \Psi+\chi)^{N}}{\mu}
$$

where $\mu$ is a volume form on $\operatorname{Hilb}_{I}^{k}(X)$. We want to show that it is positive in some open set $] 0, \lambda_{0}[\times U$ where $u \in U$. We have

$$
f(\lambda, u)=\sum_{i=0}^{i=N} C_{N}^{i} \lambda^{i} \frac{\Psi^{i} \chi^{N-i}}{\mu} .
$$

The second assumption of the lemma is that the first terms up to $i=k_{u}-1$ are non negative near $u$. Hence we have

$$
\begin{aligned}
& f(\lambda, u) \geq \sum_{i=k_{u}}^{i=N} C_{N}^{i} \lambda^{i} \frac{\Psi^{i} \chi^{N-i}}{\mu} \\
& =\lambda^{k_{u}} \sum_{i=k_{u}}^{i=N} C_{N}^{i} \lambda^{i-k_{u}} \frac{\Psi^{i} \chi^{N-i}}{\mu} .
\end{aligned}
$$

On the other hand, by the first assumption, we know that the function

$$
g(\lambda, u)=\sum_{i=k_{u}}^{i=N} C_{N}^{i} \lambda^{i-k_{u}} \frac{\Psi^{i} \chi^{N-i}}{\mu}
$$

is strictly positive at $(0, u)$ hence in a neighbourhood $]-\epsilon, \lambda_{0}[\times U$ of of $(0, u)$. But then $f(\lambda, u) \geq \lambda^{k_{u}} g(\lambda, u)$ is strictly positive in $] 0, \lambda_{0}[\times U$.

We now prove that assumptions 1 and 2 of lemma 5 are satisfied.

Proof of the assumption 1 in lemma 5. We recall the constructions of the form $\Psi$ and $\chi$. We have the diagramm

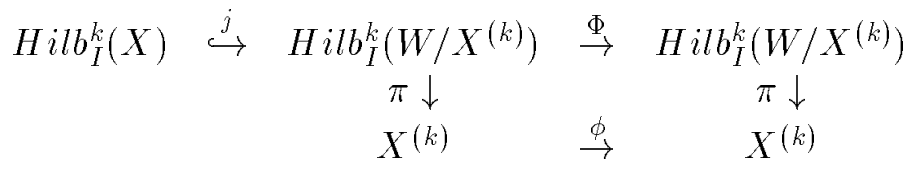

where the map $\pi$ is a fibration in complex varieties and the map $\Phi$ is holomorphic on the fibers. Then $c=\pi \circ j$ and

$$
\chi=(\phi \circ c)^{*} \omega_{k}, \Psi=(\Phi \circ j)^{*} \eta^{\prime}
$$


where $\eta^{\prime}$ is a relative Kähler form on the fibration $\pi$.

Now, if $z \in X^{(k)}$ we have the local retraction $R: X^{(k)} \rightarrow \Delta$ onto the smooth stratum containing $z$ and we can look at the diagram above locally in the charts $R \circ c: H i l b_{I}^{k}(X) \rightarrow \Delta$, using the fact that $\phi$ factors locally through the retractions $R$. The diagramm above then becomes

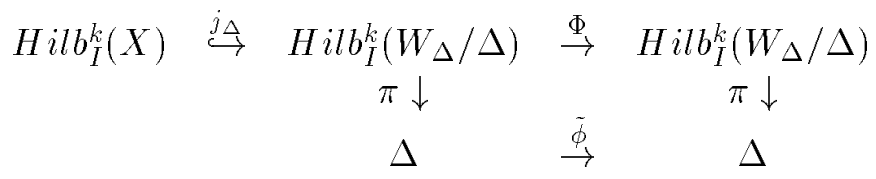

which has the main advantage that all the differentiable manifolds considered are smooth. Here we view $\Delta$ as an open set of a diagonal $\Delta_{S}$ of $X^{k}$, so that $\tilde{\phi}$ (rather than $\phi$ ) is actually defined on $\Delta$. We then have $\pi \circ j_{\Delta}=R \circ c$ and

$$
\chi=(\tilde{\phi} \circ R \circ c)^{*} \omega_{k}, \Psi=\left(\Phi \circ j_{\Delta}\right)^{*} \eta^{\prime \prime}
$$

where $\omega_{k}$ is the restriction to $\Delta$ of the form $\sum_{i} p r_{i}^{*} \omega_{i}$ and $\eta^{\prime \prime}$ is the restriction to the family $H_{i l b}^{k}\left(W_{\Delta} / \Delta\right)$ of the relative Kähler form $\eta^{\prime}$.

Recall now the local form of the map $\tilde{\phi}$ (or $\tilde{\phi}_{\mid \Delta}$ ): there exists diagonals $\Delta_{0} \subset$ $\ldots \Delta_{l} \subset \Delta$ close to $z$ such that near $z$, the relative complex structure $\tilde{I}$ factors through the retractions $R_{i}$ 's on the diagonals $\Delta_{i}$ 's and $\tilde{\phi}$ takes the form

$$
\phi_{0} \circ \ldots \circ \phi_{l}
$$

where the $\phi_{i}$ 's are the contractions onto the diagonals $\Delta_{i}$ 's given in tubular neighbourhoods by the formula (4.3).

But this last formula shows immediately that at any point $z^{\prime} \in X^{k}$, either the differential $\phi_{i, *}$ is invertible, or we have $\phi_{i}\left(z^{\prime}\right)=R_{i}\left(z^{\prime}\right)$ and $\phi_{i, *}\left(z^{\prime}\right)=R_{i, *}\left(z^{\prime}\right)$. Using the fact that the retractions $R_{i}$ 's satisfy the property $R_{j} \circ R_{i}=R_{j}$ if $\Delta_{j} \subset \Delta_{i}$, we deduce from this that at the point $z$ there is one diagonal $\Delta_{i}$ among those considered above, such that

$$
\tilde{\phi}(z) \in \Delta_{i}, \operatorname{Ker} \tilde{\phi}_{*, z}=\operatorname{Ker} R_{i, *, z}, \operatorname{Im} R_{i, *, z}=T_{\Delta_{i}, \tilde{\phi}(z)}
$$

It follows from this that for any point $u \in H_{i l b}^{k}(X)$ such that $R \circ c(u)=z$, we have

$$
\operatorname{Ker}(\tilde{\phi} \circ R \circ c)_{*, u}=\operatorname{Ker}\left(R_{i} \circ c\right)_{*, u} ., \operatorname{Im}(\tilde{\phi} \circ R \circ c)_{*, u}=T_{\Delta_{i}, \tilde{\phi}(z)}
$$

The first equation in (4.6) and the fact that $\omega_{k}$ is non degenerate on $\Delta_{i}$ implies then that

$$
\operatorname{Ker} \chi_{u}=: K_{u}=\operatorname{Ker}(\tilde{\phi} \circ R \circ c)_{*, u}=\operatorname{Ker}\left(R_{i} \circ c\right)_{*, u} .
$$

Next we note that by construction, the map $R_{i} \circ c: H i l b_{I}^{k}(X) \rightarrow \Delta_{i}$ (defined on an open set $c^{-1}(U) \subset \operatorname{Hilb}_{I}^{k}(X)$, where $U$ is a neighbourhood of $\Delta_{i}$, is a submersion whose fibers have naturally the structure of complex varieties. It follows that the vector space $K_{u}=\operatorname{Ker} R_{i} \circ c_{*, u}$ has a complex structure. We have now

Lemma 6 The form $\Psi_{u}$ restricts to a Kähler form on $K_{u}$ endowed with this complex structure. 
Assuming this we conclude as follows: it will be clear from the proof of the inequality 2 that the 2 -form $\chi_{u}$ which is equal to $\left(\psi_{i} \circ R_{i} \circ c\right)^{*} \omega^{k}$ on $T_{H i l b^{k}(X), u}$, where $\psi_{i}: T_{\Delta_{i}, R_{i}(z)} \rightarrow T_{\Delta_{i}, \tilde{\phi}(z)}$ is an diffeomorphism, induces the same orientation on $T_{H i l b^{k}(X), u} / K_{u} \cong T_{\Delta_{i}, R_{i}(z)}$ as the form $\left(R_{i} \circ c\right)^{*} \omega_{k}$. Since the orientation on $T_{H i l b^{k}(X), u}$ is compatible with the complex orientation on $K_{u}$ and the symplectic orientation on $T_{H i l b^{k}(X), u} / K_{u}$, it follows immediately that $\chi^{N-k_{u}} \Psi^{k_{u}}>0$ at $u$, which proves 1.

Proof of lemma 6. The effect of the map

$$
\Phi: \operatorname{Hilb}^{k}\left(W / X^{(k)}\right) \rightarrow H_{i l b}^{k}\left(W / X^{(k)}\right)
$$

on a fiber $(\phi \circ c)^{-1}\left(z^{\prime}\right)$ of the map $\phi \circ c: H i l b^{k}(X) \rightarrow X^{(k)}$ is the following : since the relative complex structure $I_{z}$ is equal to the complex structure $I_{z^{\prime}}$, with $z^{\prime}=\phi(z)$, and $\phi(z) \subset W_{z}$ for any $z \in X^{(k)}$, the identity map, from an open subset of $W_{z}$ to an open subset of $W_{z^{\prime}}$ containing $z$, is holomorphic with respect to the complex structures $I_{z}$ and $I_{z^{\prime}}$. Hence we can identify an open set of $H i l b_{I_{z}}^{k}\left(W_{z}\right)$ to an open set of $H i l b_{I^{\prime}}^{k}\left(W_{z^{\prime}}\right)$ and this identification is holomorphic. The effect of $\Phi$ is precisely to send

$$
(\phi \circ c)^{-1}\left(z^{\prime}\right)=\cup_{z \in \phi^{-1}\left(z^{\prime}\right)} H i l b_{I_{z}}^{k}\left(W_{z}\right) \cap c_{I_{z}}^{-1}(z)
$$

in $H i l b_{I_{z^{\prime}}}^{k}\left(W_{z^{\prime}}\right)$ via this identification.

Next assume that for some diagonal $\Delta_{j}$, a fiber $\left(c \circ R_{j}\right)^{-1}\left(z^{\prime \prime}\right)$ is contained in the fiber $(\phi \circ c)^{-1}\left(z^{\prime}\right)$; then we know that $\left(c \circ R_{j}\right)^{-1}\left(z^{\prime \prime}\right)$ has a complex structure, (being equal to $c_{I_{z^{\prime \prime}}}^{-1}\left(R_{j}^{-1}\left(z^{\prime \prime}\right)\right)$, with $R_{j}^{-1}\left(z^{\prime \prime}\right)$ a complex analytic subspace of $X^{(k)}$ for the complex structure induced by $I_{z^{\prime \prime}}$ on $\left.X^{k}\right)$, and it is obvious from the above description that via $\Phi,\left(c \circ R_{j}\right)^{-1}\left(z^{\prime \prime}\right)$ is sent holomorphically onto a complex subvariety of Hilb $_{I_{z^{\prime}}}^{k}\left(W_{z^{\prime}}\right)$.

Here we just have the infinitesimal version of thie assumption above, namely that the tangent space to the fiber $\left(c \circ R_{i}\right)^{-1}\left(z^{\prime \prime}\right), z^{\prime \prime}=R_{i} \circ c(u)$ is contained in (and even equal to) the tangent space of the fiber $(\phi \circ c)^{-1}\left(z^{\prime}\right), z^{\prime}=\phi(z)$. But the conclusion is obviously the same, namely that the inclusion

$$
\Phi_{*}: \operatorname{Ker}\left(R_{i} \circ c\right)_{*, u} \hookrightarrow T_{H_{i} b_{I_{z^{\prime}}}^{k}\left(W_{I_{z^{\prime}}}\right), \Phi(u)}
$$

is complex linear and is the inclusion of a complex subspace.

Now this is finished because the form $\eta^{\prime}$ is a relative Kähler form on the family $H i l b^{k}\left(W / X^{(k)}\right)$. Hence the form $\Psi_{u}=\Phi^{*} \eta_{\Phi(u)}^{\prime}$ restricts to a Kähler form on $K_{u}$ endowed with its complex structure.

Proof of the assumption 2 of lemma 5. We use here the notations introduced at the beginning of the section. Hence $\Delta$ is the smooth stratum of $z$, and $\Delta_{0} \subset$ $\ldots \subset \Delta_{l} \subset \Delta$ are the diagonals such that $\tilde{\phi}=\phi_{0} \circ \ldots \circ \phi_{l}$ near $z$, with $z \in V_{j}, \forall j$.

We have proved above that the form $\Psi$ restricts to a Kähler form on $K_{u}$, endowed with its complex structure coming from the identification

$$
K_{u}=\operatorname{Ker}\left(R_{i} \circ c\right)_{*, u} .
$$

In particular, since $\operatorname{Ker}(R \circ c)_{*, u}$ is a complex subspace of $\operatorname{Ker}\left(R_{i} \circ c\right)_{*, u}, \Psi$ also restricts to a Kähler form on $\operatorname{Ker}(R \circ c)_{*, u}$. Now recall formula (4.6).

$$
\chi=(\tilde{\phi} \circ R \circ c)^{*} \omega_{k} .
$$


It follows immediately that in a neighbourhood of $u, \operatorname{Ker}(R \circ c)_{*}$ is contained in the kernel of $\chi$. On the other hand, $\Psi$ gives the complex orientation on $\operatorname{Ker}(R \circ c)_{*}$. Using a local splitting of $T_{H i l b^{k}(X)}$

$$
T_{H i l b^{k}(X)}=\operatorname{Ker}(R \circ c)_{*} \oplus W
$$

(so that $W$ is naturally isomorphic to $T_{\Delta}$ ), which is orthogonal for $\Psi$, we can write in this decomposition $\Psi=\Psi_{1}+\Psi_{2}$ with $\Psi_{1}$ a Kähler form on $\operatorname{Ker}(R \circ c)_{*}$, and $\chi=\chi_{2}$. Furthermore, $W$ is isomorphic to $T_{\Delta}$ via $(R \circ c)_{*}$ and $\chi_{2}$ identifies to $\tilde{\phi}^{*} \omega_{k}$. It follows that if $i=\operatorname{dim}_{\mathbb{C}} \operatorname{Ker}(R \circ c)_{*}$ one has

$$
\Psi^{l} \chi^{N-l}=C_{l}^{i} \Psi_{1}^{i} \Psi_{2}^{l-i} \chi_{2}^{N-l} .
$$

Furthermore at any point $u^{\prime}$ close to $u$, the form $\Psi_{2}$ is close to a form $\Psi_{0}=\Psi_{u W_{u}}$ which restricts to a Kähler form on $\operatorname{Ker} \chi_{2}, u \cong \operatorname{Ker} R_{i *, z}$.

We conclude from this that it suffices to prove the following : consider the differentiable map

$$
\tilde{\phi}: \Delta \rightarrow \Delta
$$

Let $z \in \Delta$, and let

$$
K_{z}:=\operatorname{Ker} \tilde{\phi}_{*, z} \subset T_{\Delta, z} .
$$

(We know that $K_{z}=\operatorname{Ker} R_{i, *}$ is a complex subspace of $T_{\Delta, z}$ endowed with the complex structure determined by $\tilde{I}_{z}$.)

Fact.For $k=\operatorname{dim} K_{z}$ and $N=\operatorname{dim} \Delta$, and for any 2 -form $\psi$ on $T_{\Delta}$ close enough to a given form $\psi_{0}$ which restricts to a Kähler form on $K_{z}$, we have near $z$

$$
\tilde{\phi}^{*} \omega_{k}^{N-l} \psi^{l} \geq 0, \forall l \leq k_{z}=\operatorname{dim} K_{z}
$$

for the natural (symplectic) orientation of $T_{\Delta}$.

Next we recall that the map $\tilde{\phi}=\phi_{0} \circ \ldots \circ \phi_{l}$ satisfies the property

$$
R_{0} \circ \tilde{\phi}=\tilde{\phi}
$$

So $\tilde{\phi}$ acts along the fibers of the submersion $R_{0}: \Delta \rightarrow \Delta_{0}$. An argument similar as above shows now that it suffices to prove the fact above along the fibers of the map $R_{0}$, which by the explicit description of the $\phi_{i}$ 's will take the form of lemma 7 below. We have arranged that the fibers of the map $R_{0}$ identify by the tubular neighbourhoods map $i_{0}$ to a complex vector space on which the form $\omega_{k}$ restricts to a constant Kähler form. Furthermore the $R_{i}$ 's identify to linear projections. Because of the action of the symmetric group $\mathfrak{S}_{\Delta_{0}}$, preserving the form $\omega_{k}$ it is clear that these projections, which are invariant projections with respect to smaller groups, are orthogonal with respect to the Kähler form. Hence we are in the following situation.

Assume $V$ is a complex vector space of dimension $n, \omega$ is a constant Kähler form on $V$, and $h$ is the associated hermitian metric on $V$.

Let $0=V_{0} \subset \ldots \subset V_{l} \subset V$ be complex subspaces, and let $\pi_{i}: V \rightarrow V_{i}$ be the orthogonal projection. Let $\phi_{i}: V \rightarrow V$ be given by the formula

$$
\phi_{i}(v)=\mu\left(\frac{h(v)}{\eta_{i}}\right)\left(1-\pi_{i}\right)(v)+\pi_{i}(v)
$$

where the function $\mu$ is strictly increasing between $1 / 2$ and 1 and takes the value 0 in $[0,1 / 2]$ and 1 in $\left[1, \infty\left[\right.\right.$, and the $\eta_{i}$ 's are constant positive numbers. 
Let $\phi=\phi_{0} \circ \ldots \phi_{l}: V \rightarrow V$, and let $v \in V$. Let $K_{v}=\operatorname{Ker} \phi_{*, v}$ and $k_{v}=\operatorname{dim} K_{v}$. ( $K_{v}$ identifies to the kernel of some $\pi_{i}$ hence is a complex vector subspace of $T_{V, v}$.) Let $\psi_{0}$ be a 2 -form on $V$ which restrict to a Kähler form on $K_{v}$.

Lemma 7 If the $\eta_{i}$ 's satisfy $\eta_{i+1} \gg \eta_{i}, \forall i$, for any $\psi$ close enough to $\psi_{0}$, and $v^{\prime}$ close to $v$, we have

$$
\phi^{*} \omega^{n-s} \psi^{s} \geq 0, \forall s \leq k_{v}
$$

with respect to the complex orientation of $V$.

Proof. We shall content ourselves with the case where $l=1$, the general case being exactly similar. We have a decomposition

$$
V=V_{1}^{\perp} \oplus V_{1}
$$

orthogonal with respect to the Kähler form $\omega$ : with respect to this decomposition we shall write an element $z$ of $V$ as $(x, y)$. We denote by $\lambda_{i}(t)$ the function $\mu\left(t / \eta_{i}\right)$, so that

$$
\phi_{0}(x, y)=\lambda_{0}\left(|(x, y)|^{2}\right)(x, y), \phi_{1}(x, y)=\left(\lambda_{1}\left(|x|^{2}\right) x, y\right) .
$$

It is then immediate to compute

$$
\phi_{0}^{*} \omega=\lambda_{0}^{2} \omega+2 i \lambda_{0} \lambda_{0}^{\prime} \partial|z|^{2} \wedge \bar{\partial}|z|^{2} .
$$

This follows immediately from the fact that for adequate coordinates on $V_{1}$ and $V_{1}^{\perp}$, we have

$$
\omega=i\left(\sum_{j} d x_{j} \wedge d \bar{x}_{j}+\sum_{j^{\prime}} d y_{j^{\prime}} \wedge d \bar{y}_{j^{\prime}}\right),|(x, y)|^{2}=\sum_{j} x_{i} \bar{x}_{i}+\sum_{j^{\prime}} y_{j^{\prime}} \bar{y}_{j^{\prime}},
$$

and from

$$
\phi_{0}^{*} d x_{j}=\lambda_{0} d x_{j}+\lambda_{0} \lambda_{0}^{\prime} x_{i} d|z|^{2}
$$

and similarly for the $y_{j^{\prime}}$ 's. Formula (4.8) gives a splitting

$$
\omega=\omega_{x}+\omega_{y}, \omega_{x}=i\left(\sum_{j} d x_{j} \wedge d \bar{x}_{j}\right), \omega_{y}=i\left(\sum_{j^{\prime}} d y_{j^{\prime}} \wedge d \bar{y}_{j^{\prime}}\right)
$$

and exactly as before we get

$$
\phi_{1}^{*} \omega=\lambda_{1}^{2} \omega_{x}+\omega_{y}+2 i \lambda_{1} \lambda_{1}^{\prime} \partial|x|^{2} \wedge \bar{\partial}|x|^{2} .
$$

From (4.7) we deduce

$$
\phi^{*} \omega=\lambda_{0}^{2} \phi_{1}^{*} \omega+2 i \lambda_{0} \lambda_{0}^{\prime} \phi_{1}^{*}\left(\partial|z|^{2} \wedge \bar{\partial}|z|^{2}\right),
$$

and since the form $\phi_{1}^{*}\left(\partial|z|^{2} \wedge \bar{\partial}|z|^{2}\right)$ has rank 2 , we find that

$\phi^{*} \omega^{n-s}=\lambda_{0}^{2(n-s)} \phi_{1}^{*} \omega^{n-s}+2(n-s) \lambda_{0}^{\prime} \lambda_{0}^{2(n-s)-1} \phi_{1}^{*} \omega^{n-s-1} \wedge i \phi_{1}^{*}\left(\partial|z|^{2} \wedge \bar{\partial}|z|^{2}\right)$

where at $v, \lambda_{0}$ denotes $\lambda_{0}\left(\left|\phi_{1}(v)\right|^{2}\right)$ and $\lambda_{1}=\lambda_{1}\left(|v|^{2}\right)$. So it suffices to prove that near $v$

1. $\phi_{1}^{*} \omega^{n-s} \wedge \psi^{s} \geq 0, \forall s \leq k_{v}$, 
2. $\phi_{1}^{*} \omega^{n-s-1} \wedge i \phi_{1}^{*}\left(\partial|z|^{2} \wedge \bar{\partial}|z|^{2}\right) \wedge \psi^{s} \geq 0, \forall s \leq k_{v}$,

where $k=\operatorname{dim} K_{v}$, and for $\psi$ close to $\psi_{0}$ such that $\psi_{0 \mid K_{v}}$ is Kähler.

We have to distinguish three cases.

a) $\lambda_{1}$ does not vanish at $v$, and $\lambda_{0}$ does not vanish at $v$. In this case the result is obvious. Indeed, $\phi_{1}$ is then a local diffeomorphism at $v$ and similarly $\phi_{0}$ is a local diffeomorphism at $\phi_{1}(v)$ so that $\phi$ is a local diffeomorphism at $v$. Hence we have $k_{v}=0$ and we need only to prove that $\phi^{*} \omega^{n} \geq 0$. But this is obvious since by formulas (4.7) and (4.11) both $\phi_{1}$ and $\phi_{0}$ are orientation preserving at the points where they are local diffeomorphisms.

b) $\lambda_{1}$ vanishes at $v$ but $\lambda_{0}$ does not vanish. In this case we have $\phi_{1}(v)=$ $\pi_{1}(v), \phi_{1 *, v}=\pi_{1 *, v}$ and $\phi_{0}$ is a diffeomorphism near $\phi_{1}(v)$, hence

$$
K_{v}=\operatorname{Ker} \pi_{1 *, v}=V_{1}^{\perp}
$$

and the assumption is that $\psi_{0 \mid V_{1}^{\perp}}$ is Kähler.

Notice that in this case, if $\eta_{1} \ll \eta_{0}$ the second component $v_{y}$ of $v$ cannot be 0 , so that the semi-positive real $(1,1)$-form

$$
i \partial|y|^{2} \wedge \bar{\partial}|y|^{2}
$$

is non-zero at $v$.

We first prove the inequality 1 . By formula (4.11) we get

$$
\phi_{1}^{*} \omega^{n-s}=\left(\lambda_{1}^{2} \omega_{x}+\omega_{y}\right)^{n-s}+2(n-s) \lambda_{1} \lambda_{1}^{\prime}\left(\lambda_{1}^{2} \omega_{x}+\omega_{y}\right)^{n-s-1} \wedge\left(i \partial|x|^{2} \wedge \bar{\partial}|x|^{2}\right.
$$

It is enough to show that

$$
\left(\lambda_{1}^{2} \omega_{x}+\omega_{y}\right)^{n-s} \wedge \psi^{s} \geq 0,\left(\lambda_{1}^{2} \omega_{x}+\omega_{y}\right)^{n-s-1} \wedge\left(i \partial|x|^{2} \wedge \bar{\partial}|x|^{2}\right) \wedge \psi^{s} \geq 0
$$

near $v$. But each expression can be developped as a polynomial in $\lambda_{1}$. It is clear that the non-zero coefficient of smallest degree in this polynomial is equal to

$$
C_{n-s}^{k_{v}-s} \omega_{x}^{k_{v}-s} \wedge \omega_{y}^{n-k_{v}} \wedge \psi^{s}
$$

for the first expression and to

$$
C_{n-s-1}^{k_{v}-s-1} \omega_{x}^{k_{v}-s-1} \wedge \omega_{y}^{n-k_{v}} \wedge i \partial|x|^{2} \wedge \bar{\partial}|x|^{2} \wedge \psi^{s}
$$

for the second, and since $\lambda_{1}$ vanishes at $v$, it suffices to show that in each expression the non-zero coefficient of smallest degree is strictly positive at $v$ for $\psi=\psi_{0}$. But this follows immediately from the fact that the restriction $\psi_{x}$ of $\psi_{0}$ to $V_{1}^{\perp}$ is a Kähler form, and from the fact that we may assume that the component $v_{x}$ of $v$ is non zero since otherwise the function $\lambda_{1}$ is identically 0 near $v$ and the result is then obvious. Then the 2-form $i \partial|x|^{2} \wedge \bar{\partial}|x|^{2}$ is semi-positive non zero at $v$ and we have

$$
\begin{gathered}
\omega_{x}^{k_{v}-s-1} \wedge \omega_{y}^{n-k_{v}} \wedge i \partial|x|^{2} \wedge \bar{\partial}|x|^{2} \wedge \psi_{0}^{s}(v)= \\
\omega_{x}^{k_{v}-s-1} \wedge \omega_{y}^{n-k_{v}} \wedge i \partial|x|^{2} \wedge \bar{\partial}|x|^{2} \wedge \psi_{x}^{s}(v)>0 \\
\omega_{x}^{k_{v}-s} \wedge \omega_{y}^{n-k_{v}} \wedge \psi_{0}^{s}(v)=\omega_{x}^{k_{v}-s} \wedge \omega_{y}^{n-k_{v}} \wedge \psi_{x}^{s}(v)>0 .
\end{gathered}
$$


We show next the inequality 2 . We note that up to a term in $\lambda_{1}$ we have

$$
\phi_{1}^{*}\left(i \partial|z|^{2} \wedge \bar{\partial}|z|^{2}\right)=i \partial|y|^{2} \wedge \bar{\partial}|y|^{2} .
$$

We do now exactly the same computation as before with $\psi^{s}$ replaced with $i \partial|y|^{2}$ $\wedge \bar{\partial}|y|^{2} \wedge \psi^{s}$, and we conclude that

$$
\phi_{1}^{*} \omega^{n-s-1} \wedge i \phi_{1}^{*}\left(\partial|z|^{2} \wedge \bar{\partial}|z|^{2}\right) \wedge \psi^{s} \geq 0, \forall s \leq k_{v}
$$

near $v$.

c) It remains to consider the case where $\lambda_{0}$ vanishes at $v$. In this case the differential $\phi_{*, v}$ vanishes so that $K_{v}=V$ and the form $\psi_{0}$ is a Kähler form on $V$. The inequality $1: \phi_{1}^{*} \omega^{n-s} \wedge \psi_{0}^{s} \geq 0, \forall s \leq n$ is then obvious since by formula (4.11) $\phi_{1}^{*} \omega$ is a semi-positive $(1,1)$-form. With a small supplementary work, one shows that this remains in fact true for $\psi$ close enough to $\psi_{0}$. It remains now to study the inequality 2: $\phi_{1}^{*} \omega^{n-s-1} \wedge i \phi_{1}^{*}\left(\partial|z|^{2} \wedge \bar{\partial}|z|^{2}\right) \wedge \psi^{s} \geq 0, \forall s \leq n$.

Recall that

$$
\phi_{1}^{*} \omega=\lambda_{1}^{2} \omega_{x}+\omega_{y}+2 i \lambda_{1} \lambda_{1}^{\prime} \partial|x|^{2} \wedge \bar{\partial}|x|^{2}
$$

and that

$$
\phi_{1}^{*}\left(\partial|z|^{2}\right)=\partial|y|^{2}+\lambda_{1}^{2} \partial|x|^{2}+\lambda_{1} \lambda_{1}^{\prime}|x|^{2} d|x|^{2} .
$$

It follows from this that

$$
\begin{aligned}
& \phi_{1}^{*} \omega^{n-s-1}=\left(\lambda_{1}^{2} \omega_{x}+\omega_{y}\right)^{n-s-1}+ 2(n-s-1)\left(\lambda_{1}^{2} \omega_{x}+\omega_{y}\right)^{n-s-2} \wedge\left(i \lambda_{1} \lambda_{1}^{\prime} \partial|x|^{2} \wedge \bar{\partial}|x|^{2}\right), \\
& \phi_{1}^{*}\left(i \partial|z|^{2} \wedge \bar{\partial}|z|^{2}\right)= i\left(\partial|y|^{2}+\lambda_{1}^{2} \partial|x|^{2}+\lambda_{1} \lambda_{1}^{\prime}|x|^{2} d|x|^{2}\right) \\
& \wedge\left(\bar{\partial}|y|^{2}+\lambda_{1}^{2} \bar{\partial}|x|^{2}+\lambda_{1} \lambda_{1}^{\prime}|x|^{2} d|x|^{2}\right) \\
&=\quad i\left(\partial|y|^{2}+\lambda_{1}^{2} \partial|x|^{2}\right) \wedge\left(\bar{\partial}|y|^{2}+\lambda_{1}^{2} \bar{\partial}|x|^{2}\right) \\
&+i \lambda_{1} \lambda_{1}^{\prime}|x|^{2}\left(\partial|y|^{2}+\lambda_{1}^{2} \partial|x|^{2}-\bar{\partial}|y|^{2}-\lambda_{1}^{2} \bar{\partial}|x|^{2}\right) \wedge d|x|^{2} .
\end{aligned}
$$

Hence we get

$$
\begin{gathered}
\phi_{1}^{*} \omega^{n-s-1} \wedge i \phi_{1}^{*}\left(\partial|z|^{2} \wedge \bar{\partial}|z|^{2}\right) \wedge \psi^{s}=\left(\lambda_{1}^{2} \omega_{x}+\omega_{y}\right)^{n-s-2} \wedge \\
{\left[i\left(\lambda_{1}^{2} \omega_{x}+\omega_{y}\right) \wedge\left(\partial|y|^{2}+\lambda_{1}^{2} \partial|x|^{2}\right) \wedge\left(\bar{\partial}|y|^{2}+\lambda_{1}^{2} \bar{\partial}|x|^{2}\right) \wedge \psi^{s}\right.} \\
+i \lambda_{1} \lambda_{1}^{\prime}|x|^{2}\left(\lambda_{1}^{2} \omega_{x}+\omega_{y}\right) \wedge\left(\partial|y|^{2}+\lambda_{1}^{2} \partial|x|^{2}-\bar{\partial}|y|^{2}-\lambda_{1}^{2} \bar{\partial}|x|^{2}\right) \wedge d|x|^{2} \wedge \psi^{s} \\
\left.+2 i(n-s-1) \lambda_{1} \lambda_{1}^{\prime} \partial|x|^{2} \wedge \bar{\partial}|x|^{2} \wedge\left(i \partial|y|^{2} \wedge \bar{\partial}|y|^{2}\right) \wedge \psi^{s}\right]
\end{gathered}
$$

We first assume that $\lambda_{1} \neq 0$ at $v$, and that $\psi=\psi_{0}$ is Kähler.

Then the first term in this sum is strictly positive, the last term is positive or 0 and the only non positive term in the developpement of the second term is

$$
i \lambda_{1} \lambda_{1}^{\prime}|x|^{2}\left(\lambda_{1}^{2} \omega_{x}+\omega_{y}\right)^{n-s-1} \wedge \psi^{s} \wedge\left(\partial|y|^{2} \wedge \bar{\partial}|x|^{2}-\bar{\partial}|y|^{2} \wedge \partial|x|^{2}\right) .
$$

But we may assume that the number $\eta_{1}$ is very small and that the function $\lambda_{1}^{\prime}\left(|x|^{2}\right)$ is not identically 0 near $v$ (otherwise the two last terms vanish identically). Since it is identically 0 near $v$ for $\left|v_{x}\right|^{2}>1 / \eta_{1}$, this forces $\left|v_{x}\right|^{2}$ to be very small. 
Similarly, since we may assume that $\lambda_{0}$ is not identically 0 near $v$ (otherwise $\phi$ is identically 0 near $v$ and the result is trivial), $v_{y}$ cannot be very small. Then noting that we can change $\eta_{1}$ without changing the supremum of the function $\lambda_{1} \lambda_{1}^{\prime}|x|^{2}$, we find easily that the term

$$
\left.\left|i \lambda_{1} \lambda_{1}^{\prime}\right| x\right|^{2}\left(\lambda_{1}^{2} \omega_{x}+\omega_{y}\right)^{n-s-1} \wedge \psi^{s} \wedge\left(\partial|y|^{2} \wedge \bar{\partial}|x|^{2}-\bar{\partial}|y|^{2} \wedge \partial|x|^{2}\right) \mid
$$

is much smaller than

$$
\left(\lambda_{1}^{2} \omega_{x}+\omega_{y}\right)^{n-s-1} \wedge i\left(\partial|y|^{2}+\lambda_{1}^{2} \partial|x|^{2}\right) \wedge\left(\bar{\partial}|y|^{2}+\lambda_{1}^{2} \bar{\partial}|x|^{2}\right) \wedge \psi_{0}^{s},
$$

at a point $v$ in a neighbourhood of which $\lambda_{1}^{\prime}$ and $\lambda_{0}$ do not vanish identically. This implies that $\phi_{1}^{*} \omega^{n-s-1} \wedge i \phi_{1}^{*}\left(\partial|z|^{2} \wedge \bar{\partial}|z|^{2}\right) \wedge \psi^{s}$ is strictly positive near such $v$ for $\psi=\psi_{0}$ hence also for any $\psi$ close $\psi_{0}$.

To conclude we consider the case where $\lambda_{1}$ also vanishes at $v$. Then we note that the function $\lambda_{1} \lambda_{1}^{\prime}|x|^{2}$ is very small, say $\leq \epsilon$ at $v$. We expand then into powers of $\lambda_{1}$ the two polynomials

$$
\begin{gathered}
\left(\lambda_{1}^{2} \omega_{x}+\omega_{y}\right)^{n-s-1} \wedge i\left(\partial|y|^{2}+\lambda_{1}^{2} \partial|x|^{2}\right) \wedge\left(\bar{\partial}|y|^{2}+\lambda_{1}^{2} \bar{\partial}|x|^{2}\right) \wedge \psi^{s} \\
\pm \epsilon\left(\lambda_{1}^{2} \omega_{x}+\omega_{y}\right)^{n-s-1} \wedge\left(\partial|y|^{2}+\lambda_{1}^{2} \partial|x|^{2}-\bar{\partial}|y|^{2}-\lambda_{1}^{2} \bar{\partial}|x|^{2}\right) \wedge d|x|^{2} \wedge \psi^{s}, \\
i(n-s-1) \partial|x|^{2} \wedge \bar{\partial}|x|^{2} \wedge\left(\lambda_{1}^{2} \omega_{x}+\omega_{y}\right)^{n-s-2} \wedge\left(i \partial|y|^{2} \wedge \bar{\partial}|y|^{2}\right) \wedge \psi^{s} .
\end{gathered}
$$

We find that for each of them, the non zero coefficient of smallest degree is strictly positive for $\psi=\psi_{0}$. Hence we conclude that it will remain strictly positive for $\psi$ close to $\psi_{0}$ and that $\phi_{1}^{*} \omega^{n-s-1} \wedge i \phi_{1}^{*}\left(\partial|z|^{2} \wedge \bar{\partial}|z|^{2}\right) \wedge \psi^{s}$ will remain positive in a neighbourhood of $v$ since $\lambda_{1}$ remains positive. Hence lemma 7 is proven, and we have shown that the inequality (2) of lemma 5 is satisfied.

By lemma 5, this concludes the proof of theorem 5 .

\section{Some remarks and questions}

The theorem proven here leads to several problems which might be of interest for symplectic and complex algebraic geometry.

We have shown that given a compact symplectic fourfold $(X, \omega)$, we can construct for each integer $k$ a compact symplectic manifold $H i l b^{k}(X)$ of dimension $4 k$. Up to isotopy it depends only of the deformation class of $\omega$, and the deformation class of the symplectic structure on $H_{i l b}(X)$ is also determined by that of $X$.

It would be interesting to understand these symplectic varieties from a more symplectic point of view. Is it for example possible to construct them by surgery starting with simpler ones? Are they new examples of symplectic manifolds (eg exhibiting new features). The algebrogeometric analogue of this is the discovery by Beauville [1] of compact irreducible hyperkähler (or holomorphically symplectic and Kähler) varieties of any even (complex) dimension, using the punctual Hilbert schemes of $K 3$-surfaces.

Another natural question is whether these manifolds, which depend canonically on our symplectic fourfold, can be used to construct new invariants of symplectic fourfolds. Indeed, we can describe the cohomology of our varieties $H i l b^{k}(X)$ starting with the cohomology of $X$, exactly in the same way as in the integrable case 
(cf. [9], [11], [13]). This is because our manifolds admit the same fibers over $X^{(k)}$ as in the complex case, and furthermore the natural stratification of $X^{(k)}$ given by the multiplicities pulls-back to a stratification of $H_{i l b}(X)$ by singular differentiable submanifolds. Hence the same argument as in [9] applies and shows that the cohomology of $H_{i l b}(X)$ is canonically isomorphic to a direct sum of tensor products of the cohomology of $X$ with shifts of degree.

The simplest example is the case of $H^{2}\left(H i l b^{k}(X), \mathbb{Z}\right)$. It contains $c^{*} H^{2}\left(X^{(k)}, \mathbb{Z}\right)$, which is equal to the direct sum

$$
H^{2}(X, \mathbb{Z}) \oplus \wedge^{2} H^{1}(X, \mathbb{Z}) .
$$

On the other hand, denoting by $\Delta=X^{(k)}-X_{0}^{(k)}$ the big diagonal of $X^{(k)}$, which is of codimension 4 in $X^{(k)}$, one sees immediately that $E=c^{-1}(\Delta)$ is a singular differentiable submanifold of real codimension 2 of $H i l b^{k}(X)$, which admits a cohomology class $[E] \in H^{2}\left(H i l b^{k}(X), \mathbb{Z}\right)$. To see this, we note that the singular locus of $E$ is of codimension at least 4 in $H_{i l b^{k}}(X)$, so that

$$
H^{2}\left(H i l b^{k}(X), \mathbb{Z}\right)=H^{2}\left(H_{i l b}^{k}(X)-\operatorname{Sing} E, \mathbb{Z}\right),
$$

and that $E-\operatorname{Sing} E$ is canonically oriented. (Notice that all the spaces considered, including $X^{(k)}$, are stratified by smooth differentiable manifolds, which gives senses to the codimensions considered above.)

Next one checks easily that this class $[E]$ is 2-divisible, that is

$$
[E]=2 \delta, \delta \in H^{2}\left(\operatorname{Hilb}^{k}(X), \mathbb{Z}\right)
$$

and one has now, using the diffeomorphism $H_{i l b^{k}}(X)-E \stackrel{c}{\cong} X^{(k)}-\Delta$

$$
H^{2}\left(H i l b^{k}(X), \mathbb{Z}\right)=c^{*} H^{2}\left(X^{(k)}, \mathbb{Z}\right) \oplus \mathbb{Z} \delta .
$$

What has been proven in the previous section is that, given a deformation class of a symplectic structure on $X$ of fixed class $[\omega] \in H^{2}(X, \mathbb{R})$, there is a well defined deformation class of symplectic forms on $\operatorname{Hilb}^{k}(X)$ of $\operatorname{class} c^{*}[\omega]_{k}-\lambda \delta, 0<\lambda<\lambda_{0}$, for some $\lambda_{0}$ sufficiently small, where $\mu_{k} \in H^{2}\left(X^{(k)}, \mathbb{R}\right)$ denotes the image of $\mu \in$ $H^{2}(X, \mathbb{R})$ via the composed map

$$
H^{2}(X, \mathbb{R}) \stackrel{\sum_{i} p r_{i}^{*}}{\rightarrow} H^{2}\left(X^{k}, \mathbb{R}\right)^{i n v} \cong H^{2}\left(X^{(k)}, \mathbb{R}\right) .
$$

Indeed, with the notations of the previous sections, the form $\Omega=\chi+\lambda \Psi$ has for class $[\omega]_{k}-\lambda \alpha \delta$ for some fixed $\alpha$. This is because the de Rham class $[\chi]$ of $\chi$ is equal to $(\phi \circ c)^{*}[\omega]_{k}$ and $\phi$ being close to the identity acts as the identity on the cohomology of $X^{(k)}$. Next recall that $\Psi$ is the pull-back via a differentiable map $H_{i l b}(X) \rightarrow H i l b^{k}\left(\mathbb{C}^{N}\right)$ of a Kähler form $\eta$ on $H_{i l b}\left(\mathbb{C}^{N}\right)$. But this differentiable map takes value in the (smooth) open set $H i l b^{k}\left(\mathbb{C}^{N}\right)_{\text {surf }}$ made of subschemes locally contained in a smooth complex surface, and it is easy to show that

$$
H^{2}\left(H i l b^{k}\left(\mathbb{C}^{N}\right)_{\text {surf } f}, \mathbb{Z}\right)=\mathbb{Z}
$$

generated as before by a class $\delta^{\prime}$ such that $2 \delta^{\prime}$ is the class of the exceptional divisor in $H i l b^{k}\left(\mathbb{C}^{N}\right)_{\text {surf } f}$. So the class of $\eta$ is equal to $-\alpha \delta^{\prime}$ for some $\alpha>0$ and to conclude it suffices to see that the pull-back of $\delta^{\prime}$ is equal to $\delta$, which is easy. 
The first potentially interesting invariant of the deformation class $\{\omega\}$ of a symplectic structure on $X$ with fixed class $[\omega]$ is the following

$$
\lambda_{k}(X, \omega)=\max K
$$

where $K$ is the set of real numbers $\lambda_{0}$ such that for any $0<\lambda<\lambda_{0}$ there is a symplectic form of class $c^{*}[\omega]_{k}-\lambda \delta$ in the same deformation class of symplectic structures on $H i l b^{k}(X)$ already constructed. We shall compute below this invariant in the case where $X$ is the projective space $\mathbb{P}^{2}$, the deformation class of symplectic form being given by the Kähler forms of class $H=c_{1}\left(\mathcal{O}_{\mathbb{P}^{2}}(1)\right)$.

Other invariants of $(X,\{\omega\})$ can be constructed using the Gromov-Witten invariants of $H_{i l b}(X)$ endowed with its symplectic structure. Indeed the Gromov-Witten invariants of $H i l b^{k}(X)$ are defined once a deformation class of symplectic form on $H_{i l b}(X)$ is fixed. They provide for each pair of integers $(g, n), n>0$, (with $n \geq 3$ if $g=0)$ and for each integral homology class $A \in H_{2}\left(H i l b^{k}(X), \mathbb{Z}\right)$ a polynomial invariant

$$
\Phi_{A, g, n}: H^{*}\left(H i l b^{k}(X), \mathbb{Q}\right)^{\otimes n} \rightarrow \mathbb{Q} .
$$

Since $H^{*}\left(H i l b^{k}(X), \mathbb{Q}\right)$ is itself a sum of copies of tensor products of $H^{*}(X, \mathbb{Q})$, the $\Phi_{A, g, n}$ provide as well polynomial invariants on $H^{*}(X)$, depending on $A \in$ $H_{2}(X, \mathbb{Z}) \oplus \wedge^{2} H_{1}(X, \mathbb{Z}) \oplus \mathbb{Z}$.

Hence we are faced to the following alternative : either the invariants above are new, that is they can be used to distinguish symplectic fourfolds for which the previously constructed invariants are equal, or they are not and one should be able to compute them in terms of older ones.

In particular, one natural question, which might be very interesting to understand even in the algebrogeometric context is the following : is it possible to compute the Gromov-Witten invariants of $\operatorname{Hilb}^{k}(X)$ as a function of the Gromov-Witten invariants of $X$ ?

Notice that in order to understand the Gromov-Witten invariants of genus $g$ of $H_{i l b}(X)$, the Gromov-Witten invariants of genus different of $g$ are obviously necessary. For example, in the complex case, a smooth genus $g$ curve $C$ on a surface $S$ provides a smooth subvariety $j: C^{(k)} \hookrightarrow H_{i l b}(X)$. Assuming $k$ is large enough so that the Brill-Noether number (predicting the dimension of the sets of $g_{k}^{1}$ 's on $C$ ) is positive or zero, there will be rational curves $D \subset C^{(k)}$, which contribute to the Gromov-Witten invariants of $C^{(k)}$, (see [2]), and if the Gromov-Witten invariants of $X$ associated to the class $[C]$ and the genus $g$ are non-zero, it is likely that the Gromov-Witten invariants in genus 0 associated with the class $j_{*}([D])$ will be nonzero.

To conclude this section, we note that the two types of invariants mentioned above are related, leading to the computation of the invariant $\lambda_{k}$ in the case of $\left(\mathbb{P}^{2}, H\right)$. The relation is the following. Assume the Gromov-Witten invariants $\Phi_{A, g, n}$ of $H i l b^{k}(X)$ are non-zero for a class $A \neq 0$. By definition, this implies that for any symplectic form $\Omega$ on $H_{i l b^{k}}(X)$ in the deformation class considered, and any almost complex structure $J$ on $\operatorname{Hilb}^{k}(X)$ compatible with $\Omega$ there exist $J$-pseudoholomorphic curves $f: C \rightarrow H i l b^{k}(X)$ of class $A$. But then we must have

$$
<A,[\Omega]>=\int_{C} f^{*} \Omega>0 .
$$


If $[\Omega]=[\omega]_{k}-\lambda \delta$ and $\left.\langle A, \delta\rangle\right\rangle 0$, this gives the inequality

$$
\lambda_{k} \leq \frac{\left\langle A,[\omega]_{k}\right\rangle}{\langle A, \delta\rangle}
$$

We apply this observation to get

Proposition 3 The invariant $\lambda_{k}\left(\mathbb{P}^{2}, H\right)$ is equal to $1 /(k-1)$.

Proof. First of all let us denote by $\lambda_{k}^{a l g}\left(\mathbb{P}^{2}, H\right)$ the largest real number such that for any $\lambda<\lambda_{k}^{a l g}\left(\mathbb{P}^{2}, H\right)$ there exists a Kähler form of class $H_{k}-\lambda \delta$ on $H i l b^{k}\left(\mathbb{P}^{2}\right)$. Since the Kähler forms are in the deformation class of symplectic forms we constructed above on $H i l b^{k}\left(\mathbb{P}^{2}\right)$, we find that for $\lambda<\lambda_{k}^{a l g}\left(\mathbb{P}^{2}, H\right)$ there exists a symplectic form on $H i l b^{k}\left(\mathbb{P}^{2}\right)$ in the deformation class considered, it follows that

$$
\lambda_{k}^{a l g}\left(\mathbb{P}^{2}, H\right) \leq \lambda_{k}\left(\mathbb{P}^{2}, H\right)
$$

(This inequality will be true as well for any Kähler surface $S$.)

Next we use the following result which is due to Catanese and Göttsche [3]

Fact. We have

$$
\lambda_{k}^{a l g}\left(\mathbb{P}^{2}, H\right) \geq \frac{1}{k-1} .
$$

For completeness, we sketch the argument of [3]. Let $Z \subset H i l b^{k}\left(\mathbb{P}^{2}\right) \times \mathbb{P}^{2}$ be the incidence subscheme. The first projection $p r_{1}: Z \rightarrow H i l b^{k}\left(\mathbb{P}^{2}\right)$ is flat and if $L$ is a line bundle on $\mathbb{P}^{2}$, one has a vector bundle of rank $k$ on $H i l b^{k}\left(\mathbb{P}^{2}\right)$ defined as

$$
\mathcal{L}_{k}=p r_{1 *}\left(p r_{2}^{*} L\right) .
$$

One has now

Lemma $8[3] c_{1}\left(\mathcal{L}_{k}\right)=[L]_{k}-\delta$, where $[L]=c_{1}(L) \in H^{2}\left(\mathbb{P}^{2}, \mathbb{Z}\right)$.

If now $L$ is $k$-generated, that is the restriction map

$$
H^{0}\left(\mathbb{P}^{2}, L\right) \rightarrow H^{0}\left(L_{\mid Z}\right)
$$

is surjective for any 0 -dimensional subscheme $Z \subset X$ of length $k$, the natural map

$$
H^{0}(L) \otimes \mathcal{O}_{\mathbb{P}^{2}} \rightarrow \mathcal{L}_{k}
$$

is surjective, hence we find that $\operatorname{det} \mathcal{L}_{k}$ is generated by sections. Now it is easy to see that $(k-1) H$ is $k$-generated on $\mathbb{P}^{2}$, hence we find that the class $(k-1) H_{k}-\delta$ is the class of a line bundle generated by sections. The same argument shows that for any positive numbers $a, b \in \mathbb{N}^{*}$ such that $a / b>k-1$ the class $a H_{k}-b \delta$ is the class of an ample divisor on $H_{i l b}\left(\mathbb{P}^{2}\right)$. Hence it is represented by a Kähler form, and we conclude that $\lambda_{k}^{\text {alg }}\left(\mathbb{P}^{2}, H\right) \geq \frac{1}{k-1}$.

So we have proved that

$$
\lambda_{k}\left(\mathbb{P}^{2}, H\right) \geq \lambda_{k}^{a l g}\left(\mathbb{P}^{2}, H\right) \geq \frac{1}{k-1} .
$$

It remains now to show the reverse inequality

$$
\lambda_{k}\left(\mathbb{P}^{2}, H\right) \leq \frac{1}{k-1} .
$$

We use for this 
Lemma 9 Let $\Delta \subset \mathbb{P}^{2}$ be a line, and let $\mathbb{P}^{1} \subset \Delta^{(k)} \cong \mathbb{P}^{k}$ be a pencil of degree $k$ on $\Delta$. Then denoting by $j: \Delta^{(k)} \hookrightarrow H_{i l b}\left(\mathbb{P}^{2}\right)$ we have

$$
\begin{gathered}
\left.<j_{*}\left[\mathbb{P}^{1}\right], H_{k}\right]>=1 \\
\left.<j_{*}\left[\mathbb{P}^{1}\right], \delta\right]>=k-1 .
\end{gathered}
$$

Proof. We may assume that the pencil is base point free hence gives a map $\phi$ : $\mathbb{P}^{1} \rightarrow \mathbb{P}^{1}$ of degree $k$. The intersection number $\left\langle j_{*}\left[\mathbb{P}^{1}\right], \delta\right]>$ is then equal to the degree of the ramification of $\phi$, which is given by Hurwitz formula.

As for the first equality, consider the quotient map

$$
q: \Delta^{k} \rightarrow \Delta^{(k)}
$$

Then we have

$$
\left.k !<j_{*}\left[\mathbb{P}^{1}\right], H_{k}\right]>=<\left[q^{-1}\left(\mathbb{P}^{1}\right)\right], q^{*} H_{k}>.
$$

But we now that

$$
q^{*} H_{k}=\sum_{i} p r_{i}^{*} H
$$

where $H=c_{1}\left(\mathcal{O}_{\Delta}(1)\right), \Delta \cong \mathbb{P}^{1}$. Next since $q^{-1}\left(\mathbb{P}^{1}\right)$ is the curve parametrizing an element of the pencil together with an ordering of the $k$-points of the corresponding divisor on $\Delta$, the map $p r_{i}: q^{-1}\left(\mathbb{P}^{1}\right) \rightarrow \Delta$ has degree $(k-1)$ ! for every $i$, so that

$$
\left.k !<j_{*}\left[\mathbb{P}^{1}\right], H_{k}\right]>=\sum_{i=1}^{i=k}(k-1) !=k !
$$

which gives $\left.<j_{*}\left[\mathbb{P}^{1}\right], H_{k}\right]>=1$.

In order to prove that $\lambda_{k}\left(\mathbb{P}^{2}, H\right) \leq \frac{1}{k-1}$ it suffices, using this lemma and the inequality (5.17), to show that the Gromov-Witten invariants in genus 0 of $H i l b^{k}\left(\mathbb{P}^{2}\right)$ associated to the class $A=j_{*}\left[\mathbb{P}^{1}\right]$ are non-zero. For this we prove

Lemma 10 1. Every curve of class $A$ is of the form $j\left(\mathbb{P}^{1}\right)$ for some line in $\mathbb{P}^{2}$ and for some pencil of degree $k$ on it.

2. The family $\mathcal{M}$ of such curves has the expected (complex) dimension

$$
d_{\text {virt }}(A)=2 k-3-<c_{1}\left(K_{H i l b^{k}\left(\mathbb{P}^{2}\right)}\right), A>.
$$

Proof. We first prove 2. We fix as before $\Delta$ and the pencil $\mathbb{P}^{1}$. Using the fact that $\Delta^{(k)} \subset H_{i l b}\left(\mathbb{P}^{2}\right)$ is the zero set of a section of the vector bundle $\mathcal{H}_{k}$ associated to the line bundle $\mathcal{O}(1)$ on $\mathbb{P}^{2}$, we can write the exact sequence

$$
0 \rightarrow T_{\Delta(k) \mid \mathbb{P}^{1}} \rightarrow T_{H i l b^{k}\left(\mathbb{P}^{2}\right) \mid \mathbb{P}^{1}} \rightarrow \mathcal{L}_{k \mid \mathbb{P}^{1}} \rightarrow 0 .
$$

We know by lemmas 8 and 9 that the term on the right has degree $1-(k-1)=-k+2$ on $\mathbb{P}^{1}$, hence

$$
-<c_{1}\left(K_{H i l b^{k}\left(\mathbb{P}^{2}\right)}\right), A>=d^{0} T_{\Delta^{(k)} \mid \mathbb{P}^{1}}-k+2 .
$$

On the other hand, since $\Delta^{(k)} \cong \mathbb{P}^{k}$,

$$
d^{0} T_{\Delta^{(k)} \mid \mathbb{P}^{1}}=k+1
$$


and we conclude that the virtual dimension $d_{v i r t}(A)$ of the family of holomorphic maps of genus 0 and class $A$ is equal to $2 k$.

On the other hand, an element of $\mathcal{M}$ is exactly determined by the data of a line $\Delta$ in $\mathbb{P}^{2}$ and a line in $\Delta^{(k)} \cong \mathbb{P}^{k}$. The line $\Delta$ depends on two parameters and the Grassmannian of lines in $\mathbb{P}^{k}$ has dimension $2 k-2$. Hence $\operatorname{dim} \mathcal{M}=2 k$. This proves 2 .

To prove 1 , let $D \subset \operatorname{Hilb}^{k}\left(\mathbb{P}^{2}\right)$ be a curve of class $A$. Since $\left\langle A, H_{k}\right\rangle=1$ one sees immediately that there is a line $\Delta \subset \mathbb{P}^{2}$ together with a pencil of degree $l \leq k$ on $\Delta$ isomorphic to a component $D^{\prime}$ of $D$ and a cycle $z_{0}$ of degree $k-l$ such that the generic scheme $\alpha_{t}$ parametrized by $D^{\prime}$ consists of the disjoint union of a member $\gamma_{t}$ of the pencil and of a subscheme of length $k-l$ which has $z_{0}$ for associated cycle, while the union $D^{\prime \prime}$ of the other components of $D$ parametrizes schemes with fixed support. Hence we get maps $f_{1}: D^{\prime} \rightarrow H_{i l b}\left(\mathbb{P}^{2}\right), f_{2}: D^{\prime} \rightarrow H i l b^{k-l}\left(\mathbb{P}^{2}\right)$ where $f_{1}$ is an isomorphism onto a pencil of degree $l$ in a line, and $f_{2}$ takes value in a fiber of the Hilbert-Chow morphism. Furthermore, if we consider the rational map

$$
\sigma: H i l b^{l}\left(\mathbb{P}^{2}\right) \times H i l b^{k-l}\left(\mathbb{P}^{2}\right) \rightarrow H i l b^{k}\left(\mathbb{P}^{2}\right)
$$

which to $\left(z_{1}, z_{2}\right)$ associates $z_{1} \cup z_{2}$, one has $\sigma \circ\left(f_{1}, f_{2}\right)=i d_{D^{\prime}}$.

Next it is easy to see that denoting $\delta_{1}$ and $\delta_{2}$ the $\delta$-classes of the Hilbert schemes $H i l b^{l}\left(\mathbb{P}^{2}\right)$ and $H i l b^{k-l}\left(\mathbb{P}^{2}\right)$ respectively, one has

$$
<\left[D^{\prime}\right], \delta>=<D^{\prime}, f_{1}^{*} \delta_{1}>+<D^{\prime}, f_{2}^{*} \delta_{2}>+m,
$$

where the integer $m$ is defined as follows: Let $Z_{1} \rightarrow D^{\prime}$ and $Z_{2} \rightarrow D^{\prime}$ be the respective pull-back via $f_{1}$ and $f_{2}$ of the incidence schemes. There is a natural morphism

$$
\left(q_{1}, q_{2}\right): Z_{1} \times D_{D^{\prime}} Z_{2} \rightarrow \mathbb{P}^{2} \times \mathbb{P}^{2}
$$

and one has $m=$ length $\left(q_{1}, q_{2}\right)^{-1}$ (Diag). One shows now easily that $m \leq d^{0} z_{0}=$ $k-l$, using the fact that $Z_{2}$ is a family of cycles with constant support and $Z_{1}$ is given by a base point free pencil on the line $\Delta$.

We already computed that $\left\langle D^{\prime}, f_{1}^{*} \delta_{1}\right\rangle=l-1$. On the other hand, because $c \circ f_{2}$ is constant, one has $d^{0} f_{2}^{*} \delta_{2} \leq 0$ with equality only if $f_{2}$ is constant. Finally we have $\left\langle D^{\prime \prime}, \delta>\leq 0\right.$ with equality only if $D^{\prime \prime}$ is empty. Hence we conclude that

$$
<[D], \delta>\leq l-1+d^{0} z_{0}=k-1
$$

and the equality implies that $f_{2}$ is constant and $D^{\prime \prime}$ is empty. But since $[D]=A$ we have the equality $\left\langle[D], \delta>=k-1\right.$. Hence $f_{2}$ is constant, that is $\alpha_{t}=$ constant $=\alpha$ and $D^{\prime \prime}$ is empty. Finally the equality in (5.18) implies also that $m=d^{0} z_{0}=$ length $(\alpha)$, and it is easy to see that this implies that $\alpha$ is supported on $\Delta$. Hence $D$ is in fact a pencil of degree $k$ in $\Delta$.

\section{References}

[1] A. Beauville. Variétés kählériennes dont la première classe de Chern est nulle, J. Differential Geometry 18 (1983) 755-782. 
[2] A. Bertram, M. Thaddeus. On the quantum cohomology of the symmetric product of an algebraic curve, math.AG/9803026.

[3] F. Catanese, L. Göttsche. $d$-very ample line bundles and embeddings of Hilbert scheme of 0-cycles, Manuscripta Math. (68) 337-341 (1990).

[4] J. Cheah. On the Cohomology of the Hilbert scheme of points, J. Alg. Geom. $5(1996), 479-511$.

[5] G. Ellingsrud, S.-A. Stromme. On the cohomology of the Hilbert schemes of points in the plane, Inventiones Math. 87 (1987),343-352.

[6] G. Ellingsrud, L. Göttsche, M. Lehn . On the cobordism class of Hilbert schemes of points on a surface, preprint.

[7] J. Fogarty. Algebraic families on an algebraic surface, Amer. J. Math. 10 (1968), $511-521$.

[8] L. Göttsche. The Betti numbers of the Hilbert scheme of points on a smooth projective surface, Math. Ann. 286, (1990),193-207.

[9] L. Göttsche, W. Soergel. Perverse sheaves and the cohomology of Hilbert schemes of smooth algebraic surfaces, Math. Ann. 296, (1993),235-245.

[10] M. Gromov. Pseudo-holomorphic curves in symplectic manifolds, Invent. Math. 82 (1985) 307-347.

[11] J. Le Potier. La formule de Göttsche, Lecture Notes to appear.

[12] D. McDuff. Blow ups and symplectic embeddings in dimension 4, Topology, Vol 30, $n^{0} 3$, pp. 409-421, 1991.

[13] H. Nakajima. Heisenberg algebra and Hilbert schemes of points on projective surfaces, Ann. Math. 145, (1997), 379-388.

[14] Y. Ruan, G. Tian. A mathematical theory of quantum cohomology, J. Diff. Geom. 42 (1995) 259-367.

[15] J. Varouchas. Kähler spaces and proper open morphisms, Math. Ann. 283 (1989), 13-52.

[16] C. Voisin. On the Hilbert scheme of points of an almost complex fourfold, Annales de 1'Institut Fourier, Tome 50 (2000), fascicule 2, 689-722. 